\title{
Downy mildew resistance induced by Trichoderma harzianum T39 in susceptible grapevines partially mimics transcriptional changes of resistant genotypes
}

Michele Perazzolli ${ }^{1 *}$, Marco Moretto ${ }^{1}$, Paolo Fontana ${ }^{1}$, Alberto Ferrarini ${ }^{2}$, Riccardo Velasco ${ }^{1}$, Claudio Moser $^{1}$, Massimo Delledonne ${ }^{2}$ and llaria Pertot ${ }^{1}$

\begin{abstract}
Background: Downy mildew, caused by Plasmopara viticola, is one of the most severe diseases of grapevine and is commonly controlled by fungicide treatments. The beneficial microorganism Trichoderma harzianum T39 (T39) can induce resistance to downy mildew, although the molecular events associated with this process have not yet been elucidated in grapevine. A next generation RNA sequencing (RNA-Seq) approach was used to study global transcriptional changes associated with resistance induced by T39 in Vitis vinifera Pinot Noir leaves. The long-term aim was to develop strategies to optimize the use of this agent for downy mildew control.

Results: More than 14.8 million paired-end reads were obtained for each biological replicate of T39-treated and control leaf samples collected before and $24 \mathrm{~h}$ after P. viticola inoculation. RNA-Seq analysis resulted in the identification of 7,024 differentially expressed genes, highlighting the complex transcriptional reprogramming of grapevine leaves during resistance induction and in response to pathogen inoculation. Our data show that T39 has a dual effect: it directly modulates genes related to the microbial recognition machinery, and it enhances the expression of defence-related processes after pathogen inoculation. Whereas several genes were commonly affected by P. viticola in control and T39-treated plants, opposing modulation of genes related to responses to stress and protein metabolism was found. T39-induced resistance partially inhibited some disease-related processes and specifically activated defence responses after $P$. viticola inoculation, causing a significant reduction of downy mildew symptoms.

Conclusions: The global transcriptional analysis revealed that defence processes known to be implicated in the reaction of resistant genotypes to downy mildew were partially activated by T39-induced resistance in susceptible grapevines. Genes identified in this work are an important source of markers for selecting novel resistance inducers and for the analysis of environmental conditions that might affect induced resistance mechanisms.
\end{abstract}

Keywords: Induced resistance, Next generation sequencing, RNA-Seq, Transcriptomics, Gene expression, Vitis vinifera, Plant-pathogen interactions

\footnotetext{
* Correspondence: michele.perazzolli@fmach.it

${ }^{1}$ IASMA Research and Innovation Centre, Fondazione Edmund Mach, Via E. Mach 1, 38010, San Michele all'Adige (TN), Italy

Full list of author information is available at the end of the article
} 


\section{Background}

Plasmopara viticola (Berk. and Curt.) Berl. and de Toni is a biotrophic oomycete that causes downy mildew in grapevine [1]. This devastating disease occurs worldwide, particularly in regions with warm and wet conditions during the growing season. P. viticola mainly infects leaves and clusters of young berries and produces oil spot lesions on the adaxial leaf surface accompanied by massive sporulation on the abaxial surface. Although downy mildew can be controlled by frequent applications of chemical fungicides, concerns about the environmental impact of pesticide overuse [2] and the development of resistant $P$. viticola populations [3] have sparked an interest in alternatives to chemical treatments.

The grapevine industry relies predominantly on Vitis vinifera, which is susceptible to downy mildew. Resistance traits have been identified in wild grapevine species, and the mechanisms of resistance to downy mildew have been characterized in resistant genotypes [1]. Transcriptomic analysis supports the view that downy mildew resistance is mainly a post-infection phenomenon [4] and highlights the importance of transcriptional reprogramming in both resistant and susceptible genotypes in response to $P$. viticola inoculation [4-8]. Transcriptional changes associated with $P$. viticola infection of susceptible grapevines have been related to a weak defence response [4] and to the establishment of a compatible interaction $[5,7,9,10]$. The response of resistant genotypes has been characterized by strong and rapid transcriptional reprogramming of processes related to defence, signal transduction, and secondary metabolism, which are either not induced or induced to a lesser extent in susceptible grapevines $[4,8,11-14]$. In particular, downy mildew resistance has been correlated with enhanced expression of genes encoding pathogenesisrelated (PR) proteins and enzymes of phenylpropanoid biosynthesis, and with specific modulation of signal transduction components and markers of hypersensitive response (HR) in resistant grapevines [4,8,11-13].

Downy mildew symptoms can be significantly reduced in susceptible grapevines by preventive application of resistance inducers, such as chitosan [15], laminarins [16-18], $\beta$-aminobutyric acid (BABA) [19,20], acibenzolarS-methyl (BTH) [21,22], and thiamine [23]. Treatments with plant extracts [24] or microbial extracts $[22,25]$ have also been found to increase grapevine resistance to downy mildew. The ascomycete Trichoderma harzianum strain T39 (T39) significantly reduces downy mildew symptoms by activating grapevine resistance both locally and systemically [21], although the molecular events responsible for resistance induction have not yet been clarified. Trichoderma spp. strains have been characterized in model systems based on their ability to induce plant resistance against pathogens $[26,27]$ by reprogramming the plant transcriptome [28-32]. Specific strains of beneficial microorganisms can improve plant performance by activating a plant-mediated defence mechanism known as induced systemic resistance (ISR) [33]. Through root or leaf interactions [21,34], beneficial microorganisms are recognized by the plant, which results in a mild but effective activation of the plant immune responses in all tissues [35]. ISR confers broad-spectrum resistance to various types of pathogens and abiotic stresses $[27,36]$ and is usually regulated by jasmonic acid (JA)- and ethylene (ET)-dependent signalling pathways [33]. Rather than directly activating plant defences, beneficial microorganisms that induce resistance usually prime the plant so that it responds more strongly upon exposure to the stress condition $[35,36]$. Primed plants display faster and/or stronger activation of the defence responses after pathogen inoculation [37]. Because plant defences are activated only when they are really needed [38], priming provides advantages in terms of energy costs for the plant [39], and it is probably evolved to save energy under pathogen-free conditions [40]. The benefits of priming outweigh its costs when disease does occur, and priming is seen as a promising strategy in modern disease management [41]. The absence of apparent energy costs associated with T39-induced resistance in grapevine suggested a priming state activation [42]. However, the molecular mechanisms underlying resistance induction have been only partially revealed $[26,43]$, and information regarding induced resistance in grapevine is particularly scarce $[20,44]$.

In this study, we used Illumina RNA-Seq analysis to characterize the global transcriptional dynamics associated with T39-induced resistance in grapevine. To the best of our knowledge, this study is the first to use highthroughput sequencing technology to investigate molecular events underlying induced resistance in plants, and it is also the first transcriptome-wide characterization of resistance induced by a beneficial microbe in a non-model plant. Our analysis revealed that the reduction of downy mildew symptoms is related to a complex transcriptional reprogramming in T39-treated plants, both before and $24 \mathrm{~h}$ after pathogen inoculation. In particular, the reaction of T39-treated plants to pathogen inoculation is associated with enhanced expression of $P$. viticola-responsive genes and specific modulation of some genes related to defence in resistant grapevines. Our study has identified genes that could be valuable as markers of ISR activation for subsequent selection of new resistance inducers with improved ability to stimulate plant defences.

\section{Results}

RNA-Seq sequencing and mapping of reads to the grapevine genome

The ability of T39 to activate local and systemic resistance processes has been previously reported in grapevine 
$[21,42]$, and leaf treatment was used to study responses to T39 treatment and pathogen inoculation in the same plant organ. Leaf samples were collected before and $24 \mathrm{~h}$ after $P$. viticola inoculation from T39-treated and control plants, and resistance induction was confirmed by phenotypic analysis (Figure 1). Four different treatments were analysed by RNA-Seq: control (C), T39-treated (T39), P. viticola-inoculated control $(\mathrm{C}+$ P.v. $)$ and $P$. viticola-inoculated T39-treated (T39+P.v.) plants. Three biological replicates of each treatment were analysed, with each biological replicate sequenced twice in separate lanes (sequencing replicate). Between 6.1 and 18.8 million paired-end reads of 100 nucleotides were obtained for each sequencing replicate, and an average of $94 \%$ of these passed the quality control test (filtered

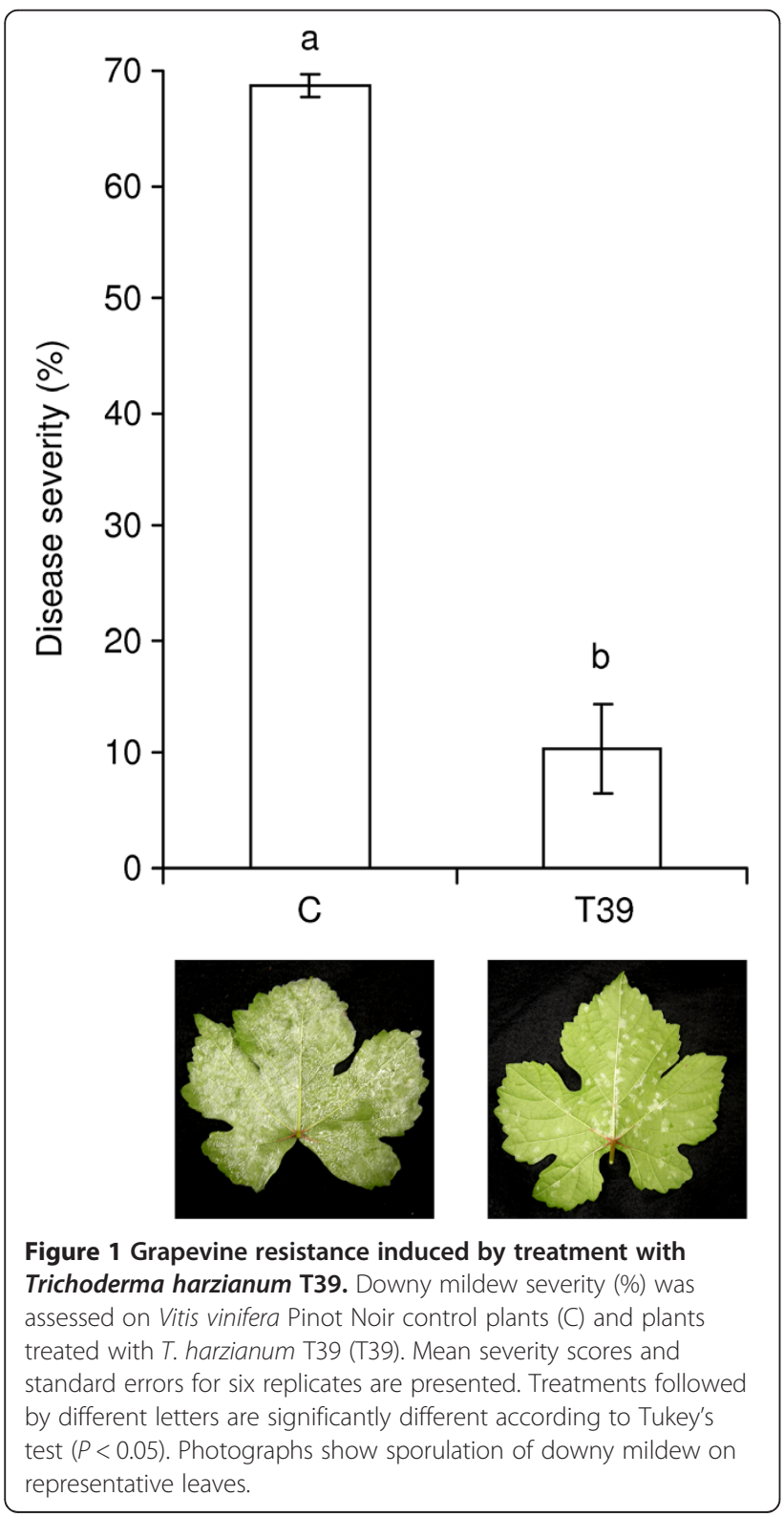

reads; Additional files 1 and 2). Summing the reads of two sequencing replicates, more than 14.8 million filtered reads were obtained for each biological replicate (Table 1), corresponding to a coverage of at least $32 \times$ the grapevine transcriptome (Table 2).

An average of $91 \%$ of filtered reads mapped to the grapevine genome (mapped reads) for each biological replicate (Table 1), and similar percentages mapped for each sequencing replicate (Additional file 1). An average of $96 \%$ of mapped reads matched to unique locations (unique reads), 4\% displayed multiple matches (multireads) to the grapevine genome (Table 1 and Additional files 1 and 3), and about $77 \%$ of mapped reads matched to grapevine genes (Additional files 3 and 4).

\section{Gene expression estimation by RNA-Seq}

Gene expression level was assessed on the basis of both unique and multi-reads (Additional file 3) to improve evaluation of members of multigene families [45,46], and it was expressed as fragments per kilobase of transcript per million fragments mapped (FPKM) values using Cufflinks [46]. Between one and nine transcript isoforms were recognized for each grapevine gene, and 3,679 to 7,548 novel genes were identified in each sequencing replicate (Additional file 5), representing important new information for genome annotation. About 66\% of grapevine genes were expressed in each sequencing replicate (Additional files 5 and 6). High correlations between sequencing replicates were obtained (Pearson's correlation coefficients and $\mathrm{R}^{2}$ values greater than 0.98 and 0.95, respectively; Additional files 7, 8 and 9), and read counts were summed to obtain better coverage and improve variance estimation of each biological replicate [47]. Considering the expression of all grapevine genes, the Pearson's correlation coefficient between control and T39-treated plants (T39 vs. C) was greater than that between control and P. viticola-inoculated plants $(\mathrm{C}+P . v$. vs. $\mathrm{C}$ and $\mathrm{T} 39+$ P.v. vs. C; Table 3), suggesting that few transcriptional changes were caused by T39 treatment and that major transcriptional reprogramming occurred after pathogen inoculation.

\section{Grapevine genes differentially expressed during Trichoderma harzianum T39-induced resistance}

DeSeq statistical analysis [47] revealed that 7,024 genes were differentially expressed with respect to the control with a false discovery rate (FDR) of $5 \%$ and a minimum fold-change of two in at least one pairwise comparison (Additional file 10). About 90\% of these genes were expressed in all treatments (Figure 2a), indicating that T39 and P. viticola caused a significant modulation (upor down-regulation greater than 2-fold) of genes normally expressed in control leaves. Relatively few genes were specifically expressed in response to the T39 
Table 1 RNA-Seq sequencing and read mapping to the grapevine genome

\begin{tabular}{|c|c|c|c|c|c|c|c|c|c|c|}
\hline Treatment $^{a}$ & Replicate $^{\text {b }}$ & Total reads ${ }^{c}$ & Filtered reads $^{d}$ & $\%$ & Mapped reads ${ }^{\mathrm{e}}$ & $\%$ & Unique reads $^{\mathrm{f}}$ & $\%$ & Multi-reads $^{9}$ & $\%$ \\
\hline \multirow[t]{3}{*}{$\mathrm{C}$} & 1 & 20480361 & 19388264 & 95 & 17975427 & 93 & 17473619 & 97 & 501808 & 3 \\
\hline & 2 & 20182309 & 19095859 & 95 & 17935380 & 94 & 17392482 & 97 & 542898 & 3 \\
\hline & 3 & 15856292 & 14845528 & 94 & 13327853 & 90 & 12749078 & 96 & 578775 & 4 \\
\hline \multirow[t]{3}{*}{ T39 } & 1 & 24312739 & 23208015 & 95 & 21103884 & 91 & 20207496 & 96 & 896388 & 4 \\
\hline & 2 & 35315408 & 32565826 & 92 & 30409086 & 93 & 29495812 & 97 & 913274 & 3 \\
\hline & 3 & 25081653 & 23722385 & 95 & 21873968 & 92 & 21145558 & 97 & 728410 & 3 \\
\hline \multirow[t]{3}{*}{ C+P.V. } & 1 & 21934228 & 20800485 & 95 & 19240139 & 92 & 18603657 & 97 & 636482 & 3 \\
\hline & 2 & 25097152 & 23684317 & 94 & 21637677 & 91 & 20896985 & 97 & 740692 & 3 \\
\hline & 3 & 22471334 & 21555054 & 96 & 19384445 & 90 & 18634940 & 96 & 749505 & 4 \\
\hline \multirow[t]{3}{*}{ T39+P.V. } & 1 & 22897386 & 21318685 & 93 & 18406407 & 86 & 17576560 & 95 & 829847 & 5 \\
\hline & 2 & 29049603 & 26710912 & 92 & 24801196 & 93 & 23981057 & 97 & 820139 & 3 \\
\hline & 3 & 22603588 & 21369934 & 95 & 19558774 & 92 & 18859902 & 96 & 698872 & 4 \\
\hline
\end{tabular}

${ }^{a}$ Grapevine leaves of control (C), Trichoderma harzianum T39-treated (T39), Plasmopara viticola-inoculated control (C+P.v.) and P. viticola-inoculated T39-treated (T39+P.v.) plants.

${ }^{\mathrm{b}}$ Biological replicates (plants), numbered from 1 to 3 .

c Total sequenced reads as sum of reads obtained from two replicates of sequencing for each treatment.

${ }^{d}$ Reads passing the quality check and the corresponding percentage (\%) of total reads.

e Reads mapping to Pinot Noir grapevine genome [77] Release 3 [78], and the corresponding percentage (\%) of filtered reads.

${ }^{f}$ Reads mapping to unique locations in the grapevine genome, and the corresponding percentage (\%) of mapped reads.

${ }^{g}$ Reads mapping to more than one location (2-100 matches) in the grapevine genome, and the corresponding percentage (\%) of mapped reads.

treatment (12 genes) and after P. viticola inoculation in control (36 genes) and T39-treated plants (22 genes).

The expression profiles of the differentially expressed genes were determined by cluster analysis, and genes were grouped into 10 clusters based on their expression modulation (Figure 2b and Additional file 10). Among

Table 2 RNA-Seq sequencing and coverage of the grapevine transcriptome for each biological replicate

\begin{tabular}{|c|c|c|c|c|}
\hline Treatment $^{a}$ & Replicate $^{\text {b }}$ & $\begin{array}{l}\text { Sequenced } \\
\text { bases (Mbp) }\end{array}$ & $\begin{array}{l}\text { Read length } \\
(\mathrm{bp})^{d}\end{array}$ & $\begin{array}{l}\text { Coverage } \\
\text { (fold) }\end{array}$ \\
\hline \multirow[t]{3}{*}{ C } & 1 & 1844 & 95 & 42 \\
\hline & 2 & 1812 & 95 & 42 \\
\hline & 3 & 1393 & 93 & 32 \\
\hline \multirow[t]{3}{*}{ T39 } & 1 & 2195 & 94 & 50 \\
\hline & 2 & 3054 & 94 & 70 \\
\hline & 3 & 2253 & 95 & 52 \\
\hline \multirow[t]{3}{*}{$C+P . V}$. & 1 & 1981 & 95 & 46 \\
\hline & 2 & 2244 & 95 & 52 \\
\hline & 3 & 2049 & 95 & 47 \\
\hline \multirow[t]{3}{*}{ T39+P.v. } & 1 & 1999 & 94 & 46 \\
\hline & 2 & 2500 & 94 & 57 \\
\hline & 3 & 2028 & 95 & 47 \\
\hline
\end{tabular}

${ }^{a}$ Grapevine leaves of control (C), Trichoderma harzianum T39-treated (T39), Plasmopara viticola-inoculated control (C+P.V.) and P. viticola-inoculated T39-treated (T39+P.V.) plants.

${ }^{b}$ Biological replicates (plants), numbered from 1 to 3 .

c Total bases (Mbp) sequenced by RNA-Seq analysis that passed the quality check.

${ }^{d}$ Mean length (bp) of sequenced reads.

e Coverage of the Pinot Noir grapevine transcriptome [77] Release

3 (43.5 Mbp) [78]. genes modulated by T39, 343 had comparable expression levels before and after $P$. viticola inoculation in T39-treated plants (cluster 1), while 233 genes were initially modulated by T39 and then further modulated by P. viticola inoculation in T39-treated plants (cluster 2). In addition, the expression of 479 genes was affected exclusively by T39 treatment and not by $P$. viticola (cluster 3). P. viticola inoculation resulted in modulation of 3,454 genes with comparable expression levels in control and T39-treated plants at $24 \mathrm{~h}$ after inoculation; of these, 948 genes were directly modulated by T39 treatment (cluster 4) and 2,506 genes were not (cluster 5). Interestingly, 868 genes showed reinforced modulation in T39-treated plants compared to control plants after $P$. viticola inoculation (ISR-primed genes), indicating

Table 3 Pearson's correlation coefficients between grapevine treatments

\begin{tabular}{ll}
\hline Comparison & $\begin{array}{c}\text { Pearson's correlation } \\
\text { coefficient }\end{array}$ \\
\hline C vs. T39 & 0.93 \\
\hline C vs. C+P.v. & 0.83 \\
\hline C vs. T39+P.v. & 0.80 \\
\hline T39 vs. C+P.v. & 0.80 \\
\hline T39 vs. T39+P.v. & 0.81 \\
\hline C+P.v. vs. T39+P.V. & 0.92 \\
\hline
\end{tabular}

Pearson's correlation coefficients are based on the expression values of all grapevine genes, expressed as fragments per kilobase of transcript per million fragments mapped (FPKM), in control (C), Trichoderma harzianum T39-treated (T39), Plasmopara viticola-inoculated control (C+P.v.), and P. viticola-inoculated T39-treated (T39+P.v.) plants. 


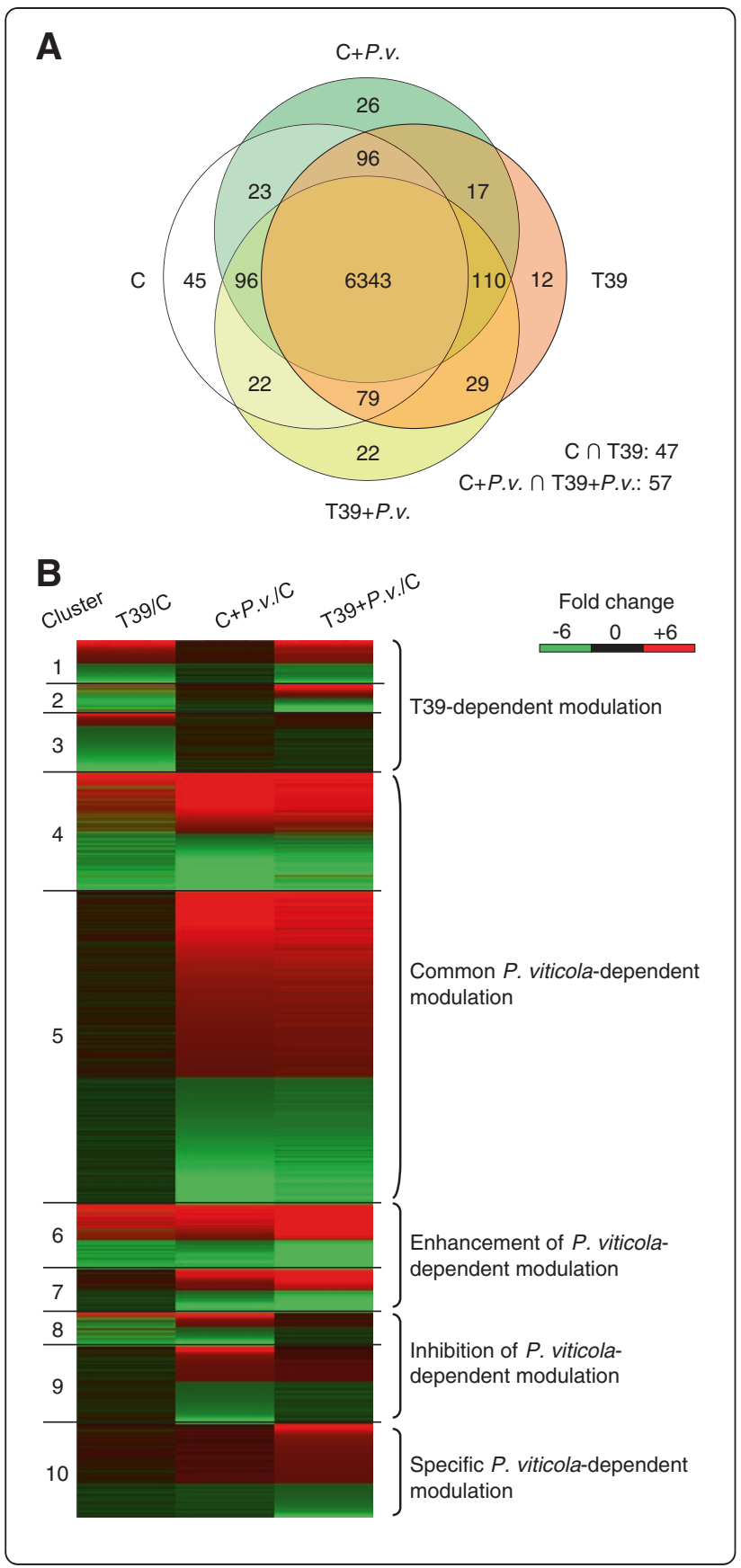

enhancement of the grapevine defence reaction to the pathogen. Among these genes, 518 and 350 genes were modulated (cluster 6) or not (cluster 7) by T39 treatment, respectively. A total of 888 genes were modulated by $P$. viticola exclusively in control plants; of these, 267 genes were directly modulated by T39 treatment (cluster 8) and 621 genes were not (cluster 9). Conversely, 759 genes were modulated by $P$. viticola exclusively in T39treated plants (cluster 10, ISR-responsive specific genes), and they represent the specific reaction to $P$. viticola of T39-treated plants. ISR-responsive specific genes
Figure 2 Grapevine genes differentially expressed during Trichoderma harzianum T39-induced resistance to Plasmopara viticola. (A) Venn diagram summarising the distribution of 7,024 differentially expressed genes in control (C), T39-treated (T39), Plasmopara viticola-inoculated control (C+P.V.) and P. viticolainoculated T39-treated (T39+P.V.) plants. Differentially expressed genes were identified by the DESeq package [47] with a false discovery rate (FDR) of $5 \%$ and a fold-change greater than two in at least one pairwise comparison. (B) Grouping of differentially expressed genes into 10 clusters based on the expression profiles. Genes modulated by T39 were distinguished as those with comparable expression levels before and after $P$. viticola inoculation in T39-treated plants (cluster 1), genes initially modulated by T39 and further modulated by P. viticola inoculation in T39-treated plants (cluster 2), and genes affected exclusively by T39 (cluster 3 ). Genes modulated by $P$. viticola with comparable expression levels in control and T39-treated plants were classified as those with (cluster 4) and without (cluster 5) direct modulation by T39. Genes modulated by $P$. viticola with greater modulation (> 1.5-fold) in T39-treated than in control plants (ISR-primed genes) where classified as those with (cluster 6) and without (cluster 7) modulation by T39 treatment. Genes modulated by $P$. viticola exclusively in control plants were classified as those with (cluster 8) and without (cluster 9) modulation by T39, whereas genes modulated by $P$. viticola exclusively in T39-treated plants were grouped in cluster 10 (ISR-responsive specific genes).

were mainly induced (63\%), while genes modulated by $P$. viticola exclusively in control plants were mainly repressed (55\%).

\section{Functional annotation of differentially expressed genes}

Differentially expressed genes were automatically annotated and then grouped into 15 functional categories of Gene Ontology (GO) biological processes (Figure 3); genes that could not be associated with any biological process category were assigned to the GO root (biological process). Although the group of genes with unknown function was the largest (35\%), 11 categories were represented to different degrees in differentially expressed genes compared to the grapevine transcriptome. DNA (5.0\%) and protein (8.4\%) metabolic processes were significantly underrepresented among the differentially expressed genes, but response to stress (5.9\%), response to stimulus (9.8\%), and signal transduction $(2.7 \%)$ categories were significantly overrepresented. Likewise, transport (6.2\%) and metabolism of carbohydrates (3.4\%), lipids (2.9\%), secondary compounds $(1.1 \%)$, and energy $(1.2 \%)$ were significantly overrepresented. The large fraction of differentially expressed genes with unknown function highlighted the identification of novel processes potentially relevant for the induction of plant resistance and for the response of grapevine to $P$. viticola inoculation. However, additional studies are required to better characterize the relevance of these genes in the mechanisms of T39-indiuced resistance. 


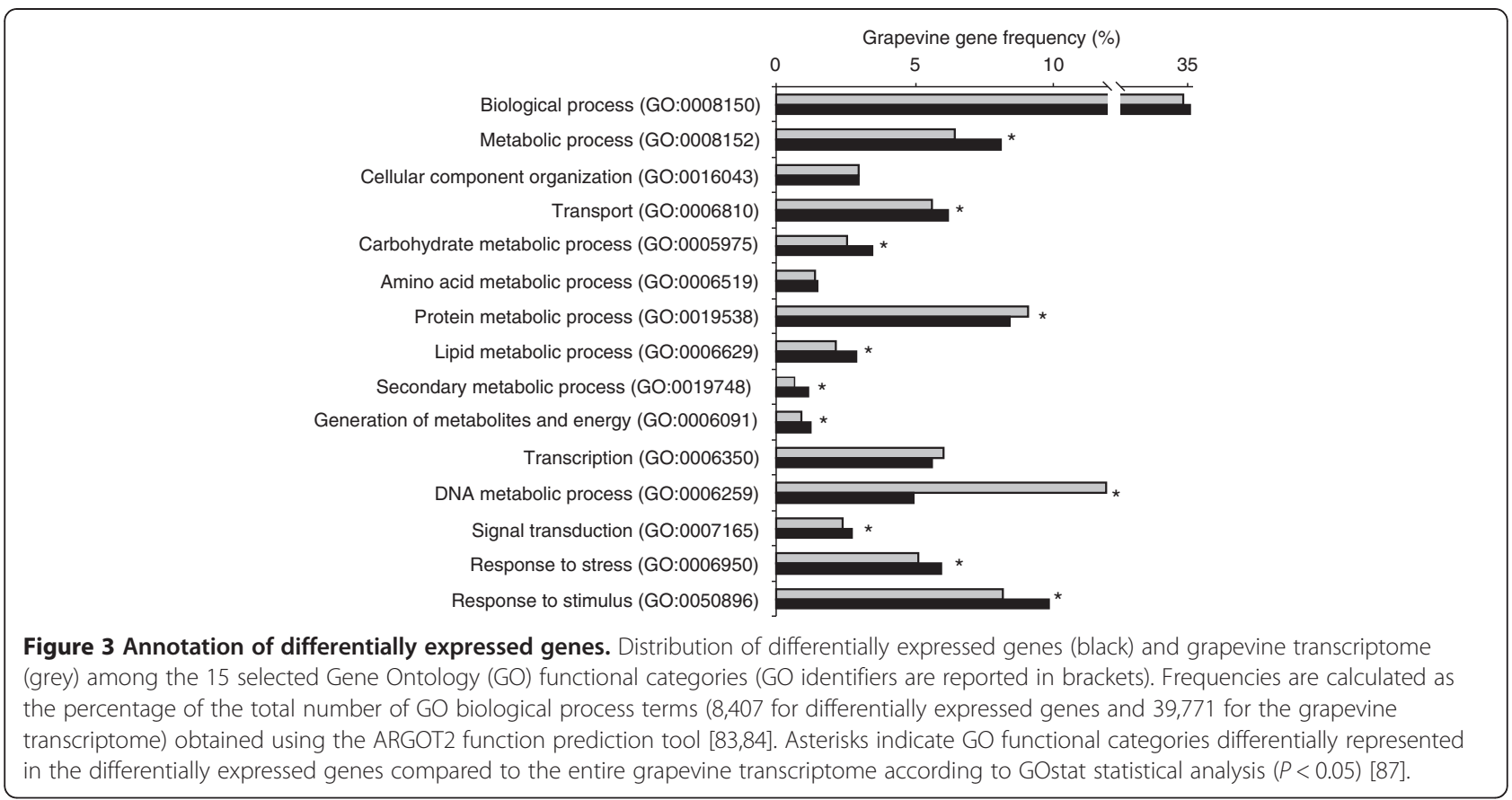

After genes with unknown function were discounted, a specific distribution of up- and down-regulated functional categories was observed in the various clusters (Figure 4). Genes of cluster 1 involved in carbohydrate, protein, and secondary metabolism were mainly induced by T39 (69, 76 and $83 \%$, respectively), whereas those related to lipid metabolism and transport were mainly repressed (86 and 65\%, respectively; Figure 4a). Genes of cluster 2 related to protein metabolism and transcription were mainly repressed by T39 and subsequently induced by $P$. viticola inoculation of T39-treated plants. More specifically, T39 treatment directly induced the expression of genes encoding protein kinases and receptor-like protein kinases, MYB transcription factors, hormoneresponsive genes, defence-related proteins, and enzymes of secondary metabolism. Annotation of genes modulated exclusively by T39 (cluster 3) revealed downregulation of processes related to response to stimulus, cellular component organization, and protein metabolism (Figure 4b).

Genes that were similarly modulated by $P$. viticola in control and T39-treated plants (clusters 4 and 5) were mainly related to primary metabolic processes and signal transduction (Figure 4c). In particular, genes involved in cellular component organization and carbohydrate metabolism were mainly induced ( 74 and $65 \%$, respectively). More specifically, $P$. viticola induced six cellulose synthase-like genes, six expansin genes, as well as genes encoding enzymes of glucan biosynthesis and hexose metabolism. Conversely, lipid metabolic process, signal transduction, response to stress, and response to stimulus were mainly repressed by $P$. viticola $(60,58,56$ and 55\%, respectively); this included genes encoding protein kinases, receptor-like protein kinases, disease resistance proteins, and PRs.

The categories of response to stress, secondary metabolism, and DNA metabolism were significantly overrepresented in clusters 6 and 7, and the genes in these categories were mainly up-regulated following $P$. viticola inoculation (Figure 4d). In particular, expression of protein kinase, disease resistance protein, chitinase (Chit), stilbene synthase (STS), resveratrol o-methyltransferase, phenylalanine ammonia-lyase (PAL), and transcription factor genes was enhanced after $P$. viticola inoculation in T39-treated plants compared with control plants.

Clusters of genes modulated by $P$. viticola exclusively in control plants revealed global down-regulation of genes related to protein metabolic process (62 and 71\% in clusters 8 and 9, respectively), response to stress (70 and 60\%, respectively), and response to stimulus (66 and 57\%, respectively; Figure 4e). Interestingly, the same categories repressed by $P$. viticola in control plants were globally induced in T39-treated plants (cluster 10; Figure 4f). In particular, genes related to protein metabolic process, response to stress, response to stimulus, and transcription were mainly up-regulated by $P$. viticola in T39-treated plants $(77,67,61$, and $67 \%$, respectively). This active reaction to the pathogen in T39-treated plants included expression of protein kinases, transcription factors, auxin and JA/ET signals, phenylpropanoid biosynthesis, and defence-related genes. 


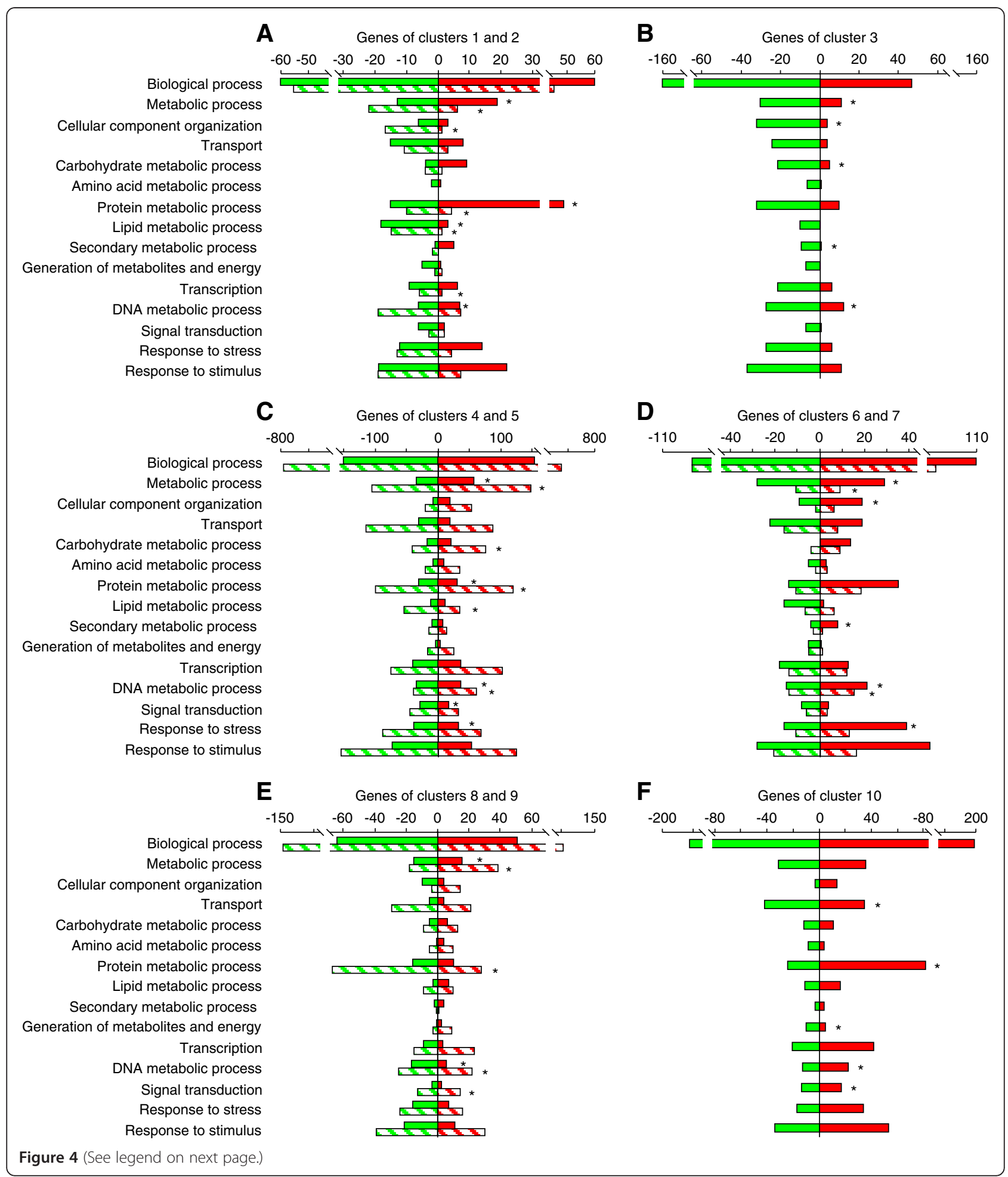

\section{Validation of RNA-Seq analysis}

To validate the RNA-Seq results, we used real-time RTPCR to analyse the expression levels of 24 differentially expressed genes (Figure 5). We selected genes with different expression profiles and expression levels, including genes associated with different functional categories (Additional file 11). Although the extent of modulation revealed by real-time RT-PCR and RNA-Seq may differ $[6,48,49]$, the real-time RT-PCR expression profiles in our analysis were in complete agreement with 
(See figure on previous page.)

Figure 4 Functional categories of differentially expressed genes grouped into clusters based on their expression profiles. Numbers of induced (red) and repressed (green) grapevine genes within each selected Gene Ontology (GO) functional category are reported for each cluster: (A) Genes modulated by Trichoderma harzianum T39 (T39) with comparable expression levels before and after Plasmopara viticola inoculation in T39-treated plants (cluster 1, solid bars) and genes initially modulated by T39 and further modulated by P. viticola inoculation in T39-treated plants (cluster 2, hatched bars); (B) Genes modulated exclusively by T39 treatment (cluster 3); (C) Genes similarly modulated by $P$. viticola in control and T39-treated plants with (cluster 4, solid bars) or without (cluster 5, hatched bars) direct modulation by T39 treatment; (D) Genes modulated by P. viticola with greater modulation (> 1.5-fold) in T39-treated than in control plants (ISR-primed genes) with (cluster 6, solid bars) or without (cluster 7, hatched bars) direct modulation by T39 treatment; (E) Genes modulated by P. viticola exclusively in control plants with (cluster 8, solid bars) or without (cluster 9, hatched bars) direct modulation by T39; (F) Genes modulated by P. viticola exclusively in T39-treated plants (cluster 10). For each cluster of differentially expressed genes, GO functional categories marked by an asterisk were differentially represented to the entire grapevine transcriptome, according to GOstat statistical analysis $(P<0.05)$ [87].

the RNA-Seq data for 19 genes. The expression profiles generated by real-time RT-PCR and RNA-Seq differed for three genes in one treatment and for two genes in all treatments. These differences could be due to differences in sensitivity, particularly in distinguishing members of multigene families. Specificity for RNA-Seq analysis was observed in two grapevine chitinases (Table 4) that are known to be strongly (Chit1a) or weakly (Chit1b) upregulated after $P$. viticola inoculation [50].

We also validated the RNA-Seq method by confirming the expression of previously identified markers of plant response to Trichoderma spp. and P. viticola (Table 4). Our RNA-Seq data confirmed $P$. viticola-dependent upregulation of $P R-1, P R-2, P R-4$, and $P R-10$ genes in susceptible grapevines $[5,7,11,19,51,52]$. Expression profiles of $P R-2, P R-4, P R-5, P R-10$, and lipoxygenase 9 (LOX-9) were consistent with previous real-time RT-PCR data obtained during T39-induced resistance [42]. The RNASeq analysis revealed T39-dependent induction of genes that are known to be involved in Trichoderma-induced resistance in other systems [26,29,53,54]. Moreover, RNA-Seq analysis confirmed the modulation of genes known to be affected by $P$. viticola in grapevines $[4-7,9,11,17,52]$.

\section{Discussion}

RNA-Seq analyses of leaf samples collected before and $24 \mathrm{~h}$ after $P$. viticola inoculation from T39-treated and control plants resulted in the identification of 7,024 differentially expressed genes. These genes formed 10 clusters of different expression profiles, highlighting the complex transcriptional reprogramming of T39-induced resistance. T39 treatment directly affected the expression of grapevine genes and to a greater extent enhanced grapevine response to $P$. viticola inoculation, indicating a dual effect of T39. At the sampling time points selected in this analysis, we showed that a limited number of changes in gene expression were caused by T39 treatment and that more intense transcriptional reprogramming took place after pathogen inoculation. In particular, opposing modulation of genes related to response to stress and protein metabolism was observed in T39-treated and control plants at $24 \mathrm{~h}$ after $P$. viticola inoculation, indicating inhibition of disease-related processes and induction of active defence in T39-treated grapevines. Based on the expression profiles, genes directly modulated by T39, as well as genes with reinforced or specific modulation in T39-treated plants after pathogen inoculation are strong candidates for activation of plant self-protection and consequent inhibition of disease-related processes and symptoms development.

\section{Grapevine processes directly affected by Trichoderma harzianum T39}

Analysis of the expression profiles revealed a set of genes directly modulated by T39 (182 up- and 161 down-regulated) and whose expression was not affected by the subsequent pathogen inoculation (cluster 1). Several genes of cluster 1 were involved in signal transduction processes, indicating that they may be related to the initial events of recognition of the beneficial microorganism and induction of resistance, as recently demonstrated by the proteomic analysis of T39-induced resistance [55]. Enzymes known to mediate microbial recognition and to trigger defence responses in plant species [56] were upregulated by T39, and these included 66 receptor-like protein kinase genes, three protein kinase genes, and one protein phosphatase gene. In particular, the serine/ threonine kinase receptor $(S T K R)$ was similar to the Arabidopsis FRK1 induced by bacterial flagellins [57], and a protein kinase was homologous to the Trichoderma-induced kinase (TIPK) of cucumber [53]. Furthermore, the STKR gene (JG391826) is induced by $P$. viticola in resistant grapevines [8], suggesting that it plays a crucial role in the activation of specific defence processes against downy mildew.

Our data suggest that resistance induction was also mediated by hormone signalling and transcriptional reprogramming. Genes related to ET metabolism (two 1-aminocyclopropane-1-carboxylate oxidases) were induced by T39 treatment, together with two $M Y B$ (including $M Y B 72$ ), one FAMA, and one NAC transcription factors. Activation of ET metabolism was in agreement with previous data showing that JA/ET signals are 


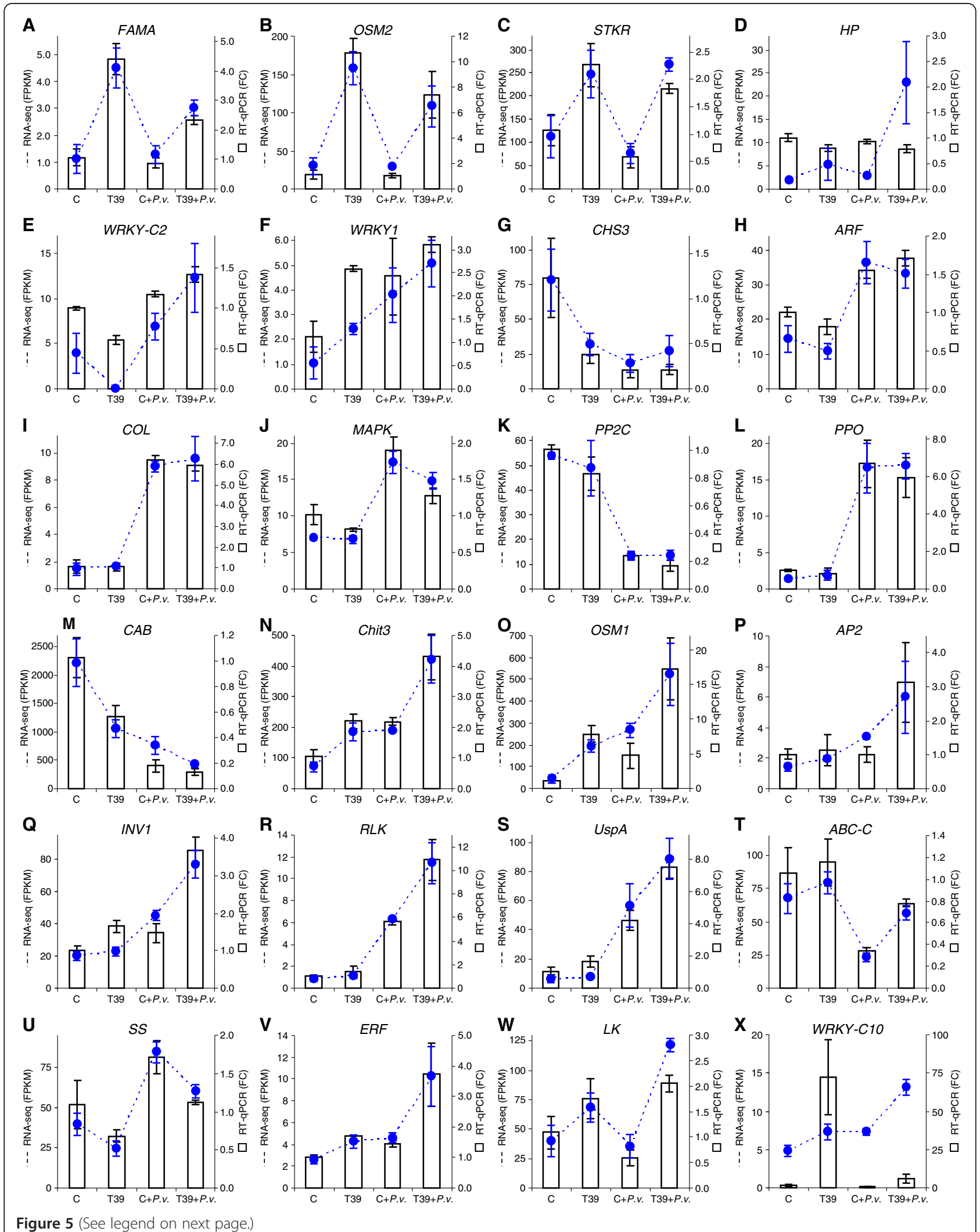


(See figure on previous page.)

Figure 5 Comparison of RNA-Seq and real-time RT-PCR analyses. Expression profiles of (A) transcription factor FAMA, (B) Trichodermainduced osmotin 2 (OSM2), (C) serine/threonine kinase receptor (STKR), (D) hypothetical protein (HP), (E) WRKY transcription factor of cluster 2 (WRKY-C2), (F) WRKY transcription factor 1 (WRKY1), (G) chalcone synthase 3 (CHS3), (H) auxin response factor (ARF), (I) constans-like protein (COL), (J) mitogen-activated protein kinase (MAPK), (K) protein phosphatase 2C (PP2C), (L) polyphenol oxidase (PPO), (M) chlorophyll a-b binding protein $(C A B),(\mathbf{N})$ acidic endochitinase 3 (Chit3), (0) Trichoderma-induced osmotin 1 (OSM1), (P) AP2-like ethylene-responsive transcription factor (AP2), (Q) invertase (INV1), (R) receptor-like serine/threonine-protein kinase (RLK), (S) universal stress A-like protein (UspA), (T) ABC transporter C family member (ABC-C), (U) sucrose synthase (SS), (V) ethylene-responsive transcription factor (ERF), (W) LRR receptor-like serine/threonine-protein kinase $(L K)$, and (X) WRKY transcription factor of cluster 10 (WRKY-C10). Blue dotted lines represent expression levels (FPKM) as assessed by RNA-Seq analysis and reported as means and standard errors of three biological replicates for each treatment: control (C), Trichoderma harzianum T39treated (T39), Plasmopara viticola-inoculated control (C+P.V.), and P. viticola-inoculated T39-treated (T39+P.V.) plants. Histograms represent the relative expression levels (fold-change relative to the expression in control plants) as assessed by real-time RT-PCR and reported as means and standard errors of three biological replicates for each treatment.

involved in T39-induced resistance [42,58]. In Arabidopsis, MYB72 plays a crucial role in Trichoderma-induced resistance [26] and is required in early signalling of rhizobacteria-induced resistance in Arabidopsis [59]. The NAC gene is involved during oxidative stress [60], and response to oxidative stress after T39 treatment was suggested by modulation of two peroxidases, three glutathione S-transferases (GSTs), and one thioredoxin. Moreover, defence-related genes (two STSs, a Chit, and a cellulose synthase) were also directly induced, indicating that the maximum expression level of these genes was probably reached after T39 treatment and that this was sufficient to contribute to defence against subsequent $P$. viticola inoculation. Other defence-related genes were pre-induced by T39 and further induced after $P$. viticola inoculation (cluster 2), indicating reinforcement of grapevine defence after pathogen inoculation.

\section{Enhanced reaction of T39-treated plants to Plasmopara viticola}

Plant resistance induced by beneficial microorganisms has been associated with faster and/or stronger activation of defence responses after pathogen inoculation [37]. P. vitiocla-responsive genes with enhanced expression in T39-treated plants were clustered to distinguish between those directly modulated (cluster 6) and those not modulated (cluster 7) by T39 treatment. These expression profiles provide strong support for the view that Trichoderma spp. may have a dual effect: it directly stimulates induction of some genes and further reinforces modulation of these and other genes after pathogen inoculation [42]. The dual effect was also reported for defencegene modulation during resistance induced by sulfated laminarin [17] and for phytoalexin accumulation during resistance induced by Rheum palmatum extracts [24].

Enhanced reaction of T39-treated plants included induction of genes related to response to stimulus and response to stress categories, suggesting improvement of signalling pathways and activation of defence reactions in response to pathogen inoculation. In particular, 50 receptor-like kinase genes (35 in cluster 6 and 15 in cluster 7) and six protein kinase genes (one in cluster 6 and five in cluster 7) of signal transduction were primed in T39-treated plants. In agreement with our results, priming was associated with increased expression of mitogen-activated protein kinases (MPK3 and MPK6) in Arabidopsis plants [43]. Up-regulation of MPK genes was also associated with the reaction of resistant grapevines to $P$. viticola $[4,6]$, and post-translational modification may be the additional mechanism for activating MPKs in response to pathogens [20]. Moreover, expression of stress-related genes was enhanced in inoculated T39-treated plants, and these genes included $P R-2, P R-4$, $P R-5, P R-10$, Chit3, and osmotin (OSM1). Similar profiles were observed for Arabidopsis $P R$ genes during $T$. asperelloides T203 and Pseudomonas syringae interactions [30], indicating common mechanisms of Trichoderma-induced resistance against biotrophs. Primed profiles were found in resistance-related genes (12 genes in cluster 6 and eight in cluster 7). Peronospora parasitica resistance genes $(R P P)$ must be expressed at optimal levels to function against downy mildew in Arabidopsis [61], and specific profiles of grapevine $R P P$ genes suggest fine tuning during T39-induced resistance.

A key role of transcriptional regulation and secondary metabolic processes in T39-induced resistance was also indicated by the priming profiles of four $M Y B$, two WRKY, and 12 ET-responsive transcription factors (e.g., $A P 2$ ), and of PAL and STSs genes. Moreover, the category DNA metabolic process was significantly overrepresented in ISR-primed genes. In particular, expression of the histone-lysine N-methyltransferases SUVR4 and $A T X 2$ was enhanced after $P$. viticola inoculation, reflecting possible involvement of epigenetic modifications [62-64] in T39-induced resistance.

Genes with enhanced expression in T39-treated plants have been previously related to defence against downy mildew in resistant grapevines. This was the case for $P R$ genes (PR-2, PR-4, PR-10, and OSM1), invertase genes (INV1 and INV2), and genes related to secondary metabolism (PAL, STSs, STKR, copper-containing amine oxidase, polyphenol oxidase, and three resveratrol 
Table 4 Expression levels of grapevine genes known to be modulated during Trichoderma spp.-induced resistance and in response to Plasmopara viticola inoculation

\begin{tabular}{|c|c|c|c|c|c|c|}
\hline \multirow[t]{2}{*}{ Gene name } & \multirow[t]{2}{*}{ Gene code $^{a}$} & \multicolumn{4}{|c|}{ Expression level (FPKM) ${ }^{\mathbf{b}}$} & \multirow[t]{2}{*}{ References $^{c}$} \\
\hline & & $\mathrm{C}$ & T39 & $C+P . v$. & $\mathrm{T} 39+P . v$. & \\
\hline Chitinase 1a (Chit1a) & glimmer.W78X092424.7_2 & 13.1 & 15.1 & 96.6 & 66.3 & Induced by P. viticola [50] \\
\hline Chitinase $1 \mathrm{~b}$ (Chit1b) & glimmer.W78X202842.8_3 & 78.4 & 213.1 & 167.2 & 340.5 & Not induced by P. viticola [50] \\
\hline Pathogenesis-related protein $1(P R-1)$ & fgenesh.W78X169899.6_1 & 262.7 & 379.7 & 770.3 & 361.9 & $\begin{array}{l}\text { Modulated during T39-induced } \\
\text { resistance in grapevine [42] }\end{array}$ \\
\hline Pathogenesis-related protein 2 (PR-2) & twinscan.W78X005385.7_1 & 214.4 & 689.8 & 604.7 & 1295.0 & $\begin{array}{l}\text { Modulated during T39-induced } \\
\text { resistance in grapevine [42] }\end{array}$ \\
\hline Pathogenesis-related protein 4 (PR-4) & glimmer.W78X053121.6_1 & 11.1 & 99.3 & 141.3 & 279.7 & $\begin{array}{l}\text { Modulated during T39-induced } \\
\text { resistance in grapevine [42] }\end{array}$ \\
\hline Pathogenesis-related protein 5 (PR-5) & glimmer.W79X000230.2_4 & 65.2 & 43.2 & 20.3 & 11.7 & $\begin{array}{l}\text { Modulated during T39-induced } \\
\text { resistance in grapevine [42] }\end{array}$ \\
\hline Pathogenesis-related protein 10 (PR-10) & sim4.W78X227342.7_2 & 1.9 & 33.9 & 14.5 & 34.8 & $\begin{array}{l}\text { Modulated during T39-induced } \\
\text { resistance in grapevine [42] }\end{array}$ \\
\hline Lipoxygenase 9 (LOX-9) & twinscan.W78X044916.31_1 & 4.3 & 6.4 & 5.0 & 10.5 & $\begin{array}{l}\text { Modulated during T39-induced } \\
\text { resistance in grapevine [42] }\end{array}$ \\
\hline Osmotin 1 (OSM1) & glimmer.W78X132476.3_2 & 43.6 & 194.6 & 268.4 & 522.1 & $\begin{array}{l}\text { Induced by T. hamatum } 382 \\
\text { in tomato }[29] \text { and induced } \\
\text { by P. viticola }[4,6]\end{array}$ \\
\hline Osmotin 2 (OSM2) & fgenesh.W78X075443.24_1 & 29.7 & 158.2 & 29.3 & 108.6 & $\begin{array}{l}\text { Induced by T. hamatum } 382 \\
\text { in tomato [29] }\end{array}$ \\
\hline $\begin{array}{l}\text { Aminocyclopropane carboxylate } \\
\text { oxidase (ACO) }\end{array}$ & fgenesh.W78X260154.9_1 & 14.8 & 40.6 & 22.1 & 36.0 & $\begin{array}{l}\text { Induced by T. koningiopsis Th003 } \\
\text { in tomato [54] }\end{array}$ \\
\hline $\begin{array}{l}\text { Aminocyclopropane carboxylate } \\
\text { oxidase (ACO) }\end{array}$ & twinscan.W78X152558.9_1 & 23.0 & 46.6 & 32.0 & 50.7 & $\begin{array}{l}\text { Induced by T. koningiopsis Th003 } \\
\text { in tomato [54] }\end{array}$ \\
\hline $\begin{array}{l}\text { Trichoderma-induced protein } \\
\text { kinase (TIPK) }\end{array}$ & fgenesh.W78X024841.39_2 & 60.6 & 226.8 & 360.3 & 374.0 & $\begin{array}{l}\text { Induced by T. asperelloides T203 } \\
\text { in cucumber [53] }\end{array}$ \\
\hline MYB transcription factor 72 (MYB72) & fgenesh.W78X183580.6_2 & 1.0 & 2.2 & 1.9 & 3.3 & $\begin{array}{l}\text { Involved in Trichoderma-induced } \\
\text { resistance [26] }\end{array}$ \\
\hline Glucanase (GLU) & glimmer.W78X010792.29_19 & 4.1 & 7.5 & 29.5 & 17.0 & Induced by P. viticola [5] \\
\hline Invertase (INV1) & fgenesh.W78X109126.4_1 & 19.9 & 22.8 & 45.1 & 76.7 & Induced by P. viticola $[4,9]$ \\
\hline Invertase (INV2) & glimmer.W78X234553.4_1 & 6.5 & 5.9 & 10.2 & 19.2 & Induced by P. viticola $[4,9]$ \\
\hline Glutathione S-transferase (GST) & glimmer.W78X070708.13_2 & 13.5 & 17.8 & 21.7 & 29.9 & Induced by P. viticola [17] \\
\hline Catalase (CAT) & glimmer.W78X186369.3_2 & 942.8 & 1519.4 & 2840.2 & 2961.4 & Induced by P. viticola [52] \\
\hline Enzymatic resistance protein (ERP) & glimmer.W78X101483.19_2 & 1078.4 & 909.1 & 435.8 & 446.9 & Repressed by P. viticola [5] \\
\hline Carbonic anhydrase $(C A)$ & fgenesh.W78X143789.26_4 & 1382.8 & 435.6 & 817.8 & 550.8 & Repressed by P. viticola [5] \\
\hline Chlorophyll a-b binding protein (CAB) & fgenesh.W78X207753.10_1 & 2220.1 & 1054.3 & 757.7 & 422.7 & Repressed by P. viticola [4] \\
\hline Dihydroflavonol 4-reductase (DFR) & glimmer.W78X173356.4_4 & 101.1 & 56.1 & 27.7 & 29.4 & Repressed by P. viticola [1 1] \\
\hline Chalcone synthase (CHS) & glimmer.W78X073177.9_2 & 55.8 & 17.3 & 6.0 & 9.5 & Repressed by P. viticola [7] \\
\hline Chalcone synthase (CHS) & glimmer.W78X195981.16_1 & 54.0 & 16.2 & 5.6 & 5.1 & Repressed by P. viticola [7] \\
\hline Chalcone synthase (CHS3) & fgenesh.W78X165120.3_1 & 78.3 & 32.1 & 18.2 & 26.8 & Repressed by P. viticola [7] \\
\hline Zeaxanthin epoxidase (ZEP) & glimmer.W78X164562.12_3 & 221.0 & 152.3 & 64.3 & 51.3 & Repressed by P. viticola [9] \\
\hline B-amylase (AMY) & fgenesh.W78X223906.2_1 & 3.7 & 3.2 & 1.4 & 1.2 & Repressed by P. viticola [10] \\
\hline B-amylase (AMY) & glimmer.W78X087147.9_2 & 57.8 & 52.7 & 11.8 & 12.0 & Repressed by P. viticola [10] \\
\hline sucrose phosphate synthase (SPS) & fgenesh.W78X130197.5_1 & 111.8 & 78.1 & 32.2 & 28.8 & Repressed by P. viticola [10] \\
\hline Phosphoglucan, water dikinase (PWD) & fgenesh.W78X092604.4_1 & 23.4 & 17.0 & 12.1 & 10.3 & Repressed by P. viticola [10] \\
\hline $\begin{array}{l}\text { Glucose-1-phosphate adenylyltransferase } \\
\text { (GPAT) }\end{array}$ & sim4.W78X153373.11_4 & 103.6 & 65.5 & 39.2 & 29.3 & Repressed by P. viticola [10] \\
\hline Threalase & fgenesh.W78X152339.9_5 & 1.0 & 1.3 & 2.7 & 2.8 & Induced by P. viticola [10] \\
\hline a,a-trehalose-phosphate synthase (TPS) & glimmer.W78X200879.5_4 & 0.3 & 0.2 & 0.1 & 0.1 & Repressed by P. viticola [10] \\
\hline Phenylalanine ammonia lyase (PAL) & glimmer.W78X178553.4_4 & 0.0 & 0.2 & 0.2 & 0.3 & Induced by P. viticola $[17,50,94]$ \\
\hline
\end{tabular}


Table 4 Expression levels of grapevine genes known to be modulated during Trichoderma spp.-induced resistance and in response to Plasmopara viticola inoculation (Continued)

\begin{tabular}{|c|c|c|c|c|c|c|}
\hline Stilbene synthase (STS) & glimmer.W78X271796.76_5 & 0.8 & 3.0 & 5.1 & 7.5 & Induced by $P$. viticola $[11,50,94]$ \\
\hline Stilbene synthase (STS) & glimmer.W78X271796.76_1 & 0.3 & 2.2 & 3.3 & 4.4 & Induced by $P$. viticola $[11,50,94]$ \\
\hline Stilbene synthase (STS) & glimmer.W78X257305.4_1 & 0.5 & 0.9 & 2.0 & 2.8 & Induced by P. viticola $[11,50,94]$ \\
\hline Stilbene synthase (STS) & glimmer.W78X121741.14_1 & 0.1 & 0.7 & 1.4 & 2.2 & Induced by $P$. viticola $[11,50,94]$ \\
\hline Amine oxidase $(A O)$ & twinscan.W78X119502.7_1 & 43.8 & 63.6 & 167.5 & 203.0 & Induced by P. viticola [8] \\
\hline Serine/threonine kinase receptor (STKR) & fgenesh.W78X255341.2_1 & 112.8 & 246.8 & 76.5 & 268.1 & Induced by P. viticola [8] \\
\hline Tropinone reductase $(T R)$ & fgenesh.W78X249214.9_1 & 0.6 & 1.0 & 12.8 & 4.1 & Induced by P. viticola [6] \\
\hline Galactinol synthase (GhGolS) & glimmer.W78X016811.4_2 & 0.1 & 0.0 & 0.6 & 0.3 & Induced by P. viticola [6] \\
\hline Wound-induced protein (WIN2) & glimmer.W78X081378.10_1 & 7.1 & 5.2 & 22.1 & 6.9 & Induced by $P$. viticola [6] \\
\hline$\beta$-glucosidase $(B G)$ & fgenesh.W78X148924.9_1 & 0.1 & 0.2 & 2.0 & 0.7 & Induced by P. viticola [6] \\
\hline TMV response-related protein (TMVR) & fgenesh.W78X106668.41_2 & 13.5 & 7.1 & 8.6 & 6.7 & Induced by P. viticola [6] \\
\hline Polygalacturonase-inhibiting protein $(P G I P)^{d}$ & glimmer.W78X089275.7_1 & - & - & - & - & Induced by P. viticola [6] \\
\hline WRKY transcription factor 21 (WRKY21) & fgenesh.W78X121610.8_2 & 32.4 & 19.9 & 6.5 & 6.9 & Induced by P. viticola [6] \\
\hline Ethylene-regulated transcript $2(E R T 2)^{d}$ & glimmer.W78X175653.7_2 & - & - & - & - & Induced by P. viticola [6] \\
\hline Rapidly elicited Avr9/Cf-9 proteins (Avr/Cf9) & fgenesh.W78X009646.12_6 & 71.5 & 70.8 & 17.9 & 21.7 & Modulated by P. viticola [5] \\
\hline Rapidly elicited Avr9/Cf-9 proteins (Avr/Cf9) & fgenesh.W78X263622.19_2 & 236.7 & 117.8 & 27.1 & 22.3 & Induced by P. viticola [4] \\
\hline Harpin-induced protein 1 (Hin 1) & glimmer.W78X225495.6_2 & 53.7 & 63.2 & 12.1 & 16.0 & Induced by P. viticola [4] \\
\hline Hypersensitivity-related 203 J (Hsr203j) & fgenesh.W78X137613.4_1 & 52.3 & 45.0 & 7.7 & 9.1 & Induced by $P$. viticola $[4,12]$ \\
\hline
\end{tabular}

${ }^{a}$ Grapevine genes of the Pinot Noir grapevine genome [77] Release 3 [78].

${ }^{\mathrm{b}}$ Gene expression levels calculated as fragments per kilobase of transcript per million fragments mapped (FPKM) in control (C), Trichoderma harzianum

T39-treated (T39), Plasmopara viticola-inoculated control (C+P.v.), and P. viticola-inoculated T39-treated (T39+P.v.) plants.

c References of previous analyses.

${ }^{\mathrm{d}}$ Genes not included in the set of differentially expressed genes.

O-methyltransferases), for which up-regulation was greater in resistant than in susceptible grapevines upon P. viticola inoculation $[4,8,11-14]$. The expression profiles of these marker genes suggest that the defence processes usually activated against downy mildew in resistant grapevines are partially stimulated in susceptible plants by T39-induced resistance.

\section{Disease-related processes inhibited in T39-treated plants} Another important aspect of T39-induced resistance was evidenced by genes modulated by $P$. viticola exclusively in control plants (clusters 8 and 9). These genes were mainly down-regulated (57 and 55\%, respectively), and they reflect exploitation of cellular resources and/ or suppression of defence responses during the compatible interaction. Down-regulation caused by $P$. viticola in control plants involved categories of response to stress and primary metabolic processes. Many signalling components (kinase, phosphatase, calmodulin, and calcium signalling), transcription factors (WRKY and $M Y B$ ), and disease resistance proteins were repressed, supporting the view that suppression of endogenous signals is required to establish the compatible interaction [5]. Three $\mathrm{ABC}$ transporters (e.g., $A B C-C$ ) were also repressed, and suppression of some $A B C$ transporters increases the susceptibility to oomycete pathogens [65]. Specific alteration of carbohydrate metabolism by
P. viticola in control plants was highlighted by modulation of glucosidase, galactosidase, mannosidase, and sucrose synthase (SS) genes. Moreover, repressed genes of cluster 9 were classified into the categories of energy metabolism (a phytochrome $\mathrm{C}$, two malic enzymes, and a ribokinase) and defence response (five chitinases, three glucanases, three superoxide dismutases, and a callose synthase), reflecting disease-related process employed by $P$. viticola only in control plants. $P$. viticola might need to actively suppress plant defences during leaf colonisation through microbial effectors, as demonstrated in other oomycetes [66,67]. Particularly, hemibiotrophic and biotrophic species establish intimate associations with plants $[66,67]$. To establish infection, these pathogens must suppress the plant defence and manipulate the host metabolism by microbial effectors (virulence factors) that are translocated inside the plant cell or secreted into the extracellular space within plant tissue $[66,68]$. P. viticola effector genes have been recently described [69], and modulation of host cell defences through virulence factors in susceptible grapevines has been indicated by histochemical [70] and transcriptomic [4] analyses. In agreement with the phenotypic observations, the specific modulation of grapevine genes in inoculated control but not in inoculated T39-treated plants indicates that T39-induced resistance acts by inhibiting some disease-related 
processes and probably by interfering with some pathogeninduced processes.

\section{Specific transcriptional response of T39-treated plants to Plasmopara viticola}

In contrast to broad down-regulation of genes in control plants (clusters 8 and 9), genes specifically modulated by P. viticola in T39-treated plants (cluster 10) were mainly induced (63\%). These opposing reactions to P. viticola are particularly evident in genes related to protein metabolism, response to stimulus, and response to stress, which were mainly induced in T39-treated plants and mainly repressed in control plants. Up-regulation of genes associated with these categories has been observed in resistant grapevines $[4,13,71]$, indicating that the defence processes of resistant genotypes could be partially activated in susceptible varieties by T39-induced resistance. In particular, all NBS-encoding resistance (NBS-R) genes modulated by $P$. viticola in control plants (cluster 9) were repressed, whereas those with ISR-responsive specific profiles (cluster 10) or ISR-primed profiles (cluster 7) were mainly up-regulated. Opposing modulation of NBS-R genes probably reflects suppression of plant defence in control plants and activation of defence responses in T39-treated plants. Interestingly, the NBS$R$ genes of clusters 7 and 10 belong mainly to the $\mathrm{Va}$ component genome of grapevine [71], indicating subgenome-dependent regulation of gene expression [72] in grapevine.

Defence signals specifically activated in T39-treated plants included those mediated by auxin (two auxin transporters, two auxin-induced proteins, and two indole-3-acetic acid amido synthetases), ET (ACC oxidase and five ERF transcription factors), and JA (three lipoxygenases and two fatty acid desaturases). The role of JA/ET signalling pathways in T39-induced resistance has also been demonstrated by phytohormone-affected Arabidopsis mutants [58] and by expression analysis of grapevine marker genes [42]. ISR is commonly regulated by JA/ET-dependent signalling pathways, and it is especially active against pathogens deterred by defences that are controlled by JA and ET $[33,36]$. The auxin response pathway is connected to the SA and JA/ET signalling networks [33], and crosstalk between hormonal pathways [36] enables the fine tuning of defence mechanisms so that the plant can tailor its response to the specific invader [33]. ET exerts its resistance-stimulating activity in concert with JA [33], and JA pathways are involved in the reaction to $P$. viticola in resistant grapevines $[4,6]$. Thus, enhancement of JA/ET signals in T39-treated plants supports the view that increased resistance to downy mildew is mediated by partial activation of extant defence mechanisms normally activated in resistant genotypes. Reaction to the pathogen was also mediated by specific up-regulation of 59 receptor kinases, 10 protein kinases, two bHLHs genes, one MYB gene, and the NPR1.1 gene.

Our results also suggest that the cell redox balance is altered in T39-treated plants after pathogen inoculation. The reaction of T39-treated plants to pathogen inoculation included the induction four peroxidases and a GST. Antioxidant enzymes are often induced in response to pathogens, and alteration of oxidative-stress metabolism has a prominent role in the T39-induced resistance of grapevine to downy mildew [55]. Peroxidases play several important roles in pathogen resistance by contributing to the production of reactive oxygen species, the reinforcement of cell walls, and the production of phytoalexins. Accumulation of stilbene phytoalexin is one of the most important defence processes activated by resistant grapevines in response to P. viticola [1]; genes of phenylpropanoid biosynthesis (flavanone 3-dioxygenase, laccase, and dihydroflavonol-4-reductase) were specifically induced in T39-treated plants, confirming activation of pathways known in resistant genotypes. However, additional defence mechanisms against $P$. viticola are activated in resistant genotypes. In particular, HR-related genes (Avr/Cf9, Hin1, and Hsr203j) were not induced and localised HR necrosis was not observed in T39treated plants.

\section{Common transcriptional response of control and T39-treated plants to Plasmopara viticola}

Although specific transcriptional reprogramming of T39-treated plants was observed, 3,454 genes had comparable expression levels in control and T39-treated plants after $P$. viticola inoculation (clusters 4 and 5). The pathogen-responsive processes not affected by resistance induction were mainly related to primary metabolism and signal transduction. In particular, expression profiles of genes related to starch metabolism (up-regulation of $\alpha$-amylase and sugar transporters, down-regulation of $\beta$-amylase, glucose-1-phosphate adenylyltransferases, sucrose phosphate synthase, and phosphoglucan water dikinase) indicated the source-to-sink transition of $P$. viticola-infected leaves [10]. Likewise, genes related to photosynthesis (two quinone oxidoreductases, a chlorophyllide oxygenase, a protochlorophyllide transporter, a chlorophyllase-2 and chlorophyll a-b binding proteins) and to the Calvin cycle (two rubisco genes and a phosphoglycerate kinase gene) were similarly modulated in inoculated control and T39-treated plants, possibly reflecting the establishment of a compatible interaction. $P$. viticola inoculation also resulted in the down-regulation of genes involved in the signal transduction processes (20 receptor-like protein kinase genes, 11 protein kinase genes, and five protein phosphatase genes) and defence response (23 probable 
disease resistance genes, six unspecified $P R$ genes, a GST gene and a thaumatin-like gene [73]), indicating a pathogen-dependent suppression of the host reaction mechanisms.

Other markers of $P$. viticola infection in susceptible grapevines showed comparable modulation in T39-treated and control plants, such as enzymatic resistance protein [5], zeaxanthin epoxidase [9], catalase [52], isoforms of chalcone synthase [7], and dihydroflavonol 4-reductase [11]. These results suggested that transcriptional changes associated with the compatible interaction $[5,7,10,13]$ were not completely inhibited in T39-treated plants, which is consistent with the observation that downy mildew symptoms were reduced but not completely blocked by T39 treatment.

\section{Conclusions}

The transcriptome analysis reported here represents a major contribution to the characterization of induced resistance mechanisms in a non-model plant. We used the RNA-Seq approach to characterize the transcriptional changes associated with the reduction of downy mildew symptoms in T39-treated grapevines and thereby established a foundation for a more detailed time-course analysis of induced resistance in grapevines. Our data show that T39 directly activates microbial recognition mechanisms in absence of pathogen infection and enhances the expression of defence processes after $P$. viticola inoculation. Reduction of downy mildew symptoms is related to inhibition of disease-related processes and activation of defence mechanisms after P. viticola inoculation of T39-treated plants. T39-induced resistance is associated with enhanced expression of specific genes related to resistance against downy mildew in wild grapevines, indicating that induced resistance can partially mimic defence processes of resistant genotypes. In addition, the genes identified in this work represent an important source of markers of resistance induction and can be used to select beneficial microorganisms with an improved ability to induce plant resistance. These markers can also be used to clarify how environmental conditions affect induced resistance.

\section{Methods}

\section{Biological materials}

Two-year-old plants of the susceptible grapevine ( $V$. vinifera) cultivar Pinot Noir grafted onto Kober 5BB were individually planted in $2.5 \mathrm{~L}$ pots containing a mixture of peat and pumice $(3: 1)$. Plants were grown for two months in a greenhouse at $25 \pm 1{ }^{\circ} \mathrm{C}$ with a photoperiod of $16 \mathrm{~h}$ light and relative humidity (RH) of $70 \pm 10 \%$. $T$. harzianum T39 (Trichodex, Makhteshim Ltd., Israel) was applied to grapevine leaves at $8 \mathrm{~g} \mathrm{~L}^{-1}$ in water, corresponding to a conidial suspension of $10^{5}$ colony-forming units (cfu) $\mathrm{mL}^{-1}$. A P. viticola isolate was collected from an untreated vineyard in the Trentino region (northern Italy) and maintained by subsequent inoculations on $V$. vinifera Pinot Noir plants under controlled greenhouse conditions. Plants with oil spot symptoms were incubated overnight in the dark at 99-100\% RH. $P$. viticola sporangia were collected by washing the abaxial surfaces bearing freshly sporulating lesions with cold distilled water, and the inoculum concentration was adjusted to $10^{5}$ sporangia $\mathrm{mL}^{-1}$ with a haemocytometer.

\section{Bioassay of induced resistance in grapevine}

All leaves of each shoot were treated three times with T39 (at one, two and three days before pathogen inoculation) to induce the greatest phenotypic resistance response [21]. Leaves directly treated with the resistance inducer were analysed to study local effects of T39induced resistance, and untreated plants were used as control. The suspensions were applied to the abaxial and adaxial leaf surfaces using a compressed air hand sprayer (20-30 mL for each plant).

One day after the last T39 treatment, the abaxial surfaces of all leaves of each plant were inoculated with a $P$. viticola suspension $\left(10^{5}\right.$ sporangia $\left.\mathrm{mL}^{-1}\right)$ using a compressed-air hand sprayer $(20-30 \mathrm{~mL}$ for each plant). All plants were then incubated overnight in the dark at $25 \pm 1{ }^{\circ} \mathrm{C}$ with $99-100 \% \mathrm{RH}$ and then kept under controlled greenhouse conditions.

Six days after inoculation with the pathogen, all plants were incubated overnight in the dark at $25^{\circ} \mathrm{C}$ with 99$100 \%$ RH. Disease severity was visually assessed as the percentage of abaxial leaf area covered by sporulation [74]. Each treatment was carried out on six replicates (plants). An ANOVA analysis was performed using the Statistica 9 software (StatSoft, Tulsa, OK) followed by Tukey's test to detect significant differences $(P<0.05)$ in disease severity between treatments.

\section{Sample collection and RNA isolation}

Leaf samples were collected from control and T39treated plants immediately before and $24 \mathrm{~h}$ after $P$. viticola inoculation. This time point was chosen because it is associated with leaf colonization by primary hyphae $[24,55,75]$ and with modulation of defence-related genes $[4,17,19,42]$ for the establishment of defence responses in resistant genotypes [4]. Four treatments were analysed by RNA-Seq: control (C), T39-treated (T39), P. viticolainoculated control (C+P.v.), and P. viticola-inoculated T39-treated (T39+P.v.) plants. For each treatment, leaf samples from three replicates (plants) were collected at each time point, i.e., three plants per treatment were sampled before inoculation and three plants per treatment were sampled $24 \mathrm{~h}$ after inoculation; different plants were sampled at each time point to avoid the 
effects of wounding stress. Each sample comprised three leaves taken from the same plant, and only leaves of the $4^{\text {th }}-5^{\text {th }}$ node from the top of the shoot were collected to avoid ontogenic resistance effects [42]. Samples were immediately frozen in liquid $\mathrm{N}_{2}$ and stored at $-80^{\circ} \mathrm{C}$. Total RNA was extracted using the Spectrum Plant total RNA kit (Sigma-Aldrich, St. Louis, MO) and quantified using the Nanodrop 8000 (Thermo Fisher Scientific, Wilmington, $\mathrm{DE})$. The quality of the RNA extracts was checked using the Bioanalyzer 2100 (Agilent Technologies, Santa Clara, CA).

\section{Library construction and Illumina sequencing}

Three biological replicates for each treatment were subjected to RNA-Seq library construction, and each library was sequenced twice in separate lanes (sequencing replicate). In a preliminary experiment, a technical replicate of one sample was run, and the results showed that the library preparation had a high level of technical reproducibility $\left(R^{2}=0.93\right.$, Pearson's correlation $\left.=0.96\right)$. Libraries were prepared using the TruSeq SBS v5 protocol (Illumina, San Diego, CA), and paired-end reads of 100 nucleotides were obtained using an Illumina HiSeq 2000 at Fasteris (Plan-les-Ouates, Switzerland). Briefly, poly (A)-containing mRNA was isolated from $10 \mu \mathrm{g}$ of total RNA in two rounds of purification using poly-T oligoattached magnetic beads. Purified mRNA was fragmented using Zn-catalysed hydrolysis and converted into double-stranded cDNA by random priming. Following end repair, indexed adapters were ligated and cDNA fragments of $200 \pm 25$ bp were purified. Purified cDNA was amplified by PCR and validated by Sanger sequencing, after which mRNA-Seq libraries were multiplexed (six libraries per lane) and sequenced according to the manufacturer's instructions. The sequences have been deposited at the Sequence Read Archive of the National Center for Biotechnology [76] under BioProject number PRJNA168987.

\section{Mapping of sequenced reads and assessment of gene expression}

For sequence quality control, in-house python scripts were used for quality trimming and sequence filtering: sequencing adapters, k-mers, and bases with a Phred quality score lower than 30 were removed from the read ends, and reads shorter than 50 nucleotides were then discarded. Filtered reads were mapped to the Pinot Noir grapevine genome [77] Release 3 [78] using TopHat 1.2.0 release with default settings [79]; up to 100 hits for each read were allowed to improve gene expression evaluation of members of multigene families [45]. Gene expression values were determined using Cufflinks 1.0.3 release [46], and the FPKM values were calculated for each transcript. Cufflinks default settings were adopted and gene FPKM values were computed by summing the FPKM values of different transcripts of the same gene [46]. The option for fragment bias correction was applied [80], and the RABT assembly option was used to improve the identification of novel genes and transcripts [81]. Sequencing replicates were first analysed separately, and then the read counts of two sequencing replicates were summed and FPKM values recalculated for each biological replicate.

\section{Differential gene expression analysis}

The number of reads falling into each transcript was estimated from the FPKM values according to the formula reported by Zenoni et al. [49], and gene counts were computed by summing counts of different transcripts of the same gene [46]. Differentially expressed genes were identified by the DESeq package [47], with an FDR of the Benjamini-Hochberg multiple tests of $5 \%$ $(P<0.05)$ based on read counts and a minimum foldchange of two in at least one pairwise comparison: T39 versus $\mathrm{C}, \mathrm{C}+$ P.v. versus $\mathrm{C}$, or $\mathrm{T} 39+P . v$. versus $\mathrm{C}$. Foldchanges were calculated from FPKM expression values with the minimum expression value $\left(10^{-4}\right.$ FPKM) imposed on non-expressed genes. Genes modulated by pathogen inoculation exclusively in T39-treated plants were classified as ISR-responsive specific genes [38]. Among genes modulated by $P$. viticola in control and T39-treated plants, genes that showed an up- or downregulation greater than 1.5 -fold in inoculated T39treated versus inoculated control plants were selected as ISR-primed genes. This priming cut-off value is based on previous expression data of Arabidopsis genes that showed a primed expression profile after pathogen attack [38]. ISR-primed genes were further distinguished as genes directly modulated (cluster 6) or not (cluster 7) by T39 treatment. Principal component analysis (PCA) was performed with Statistica 9 software (StatSoft, Tulsa, OK) on FPKM expression values of all grapevine genes, and the expression profiles of differentially expressed genes were determined by cluster analysis using the T-MeV 4.8.1 software [82].

\section{Gene annotation}

The grapevine genes were automatically annotated using the ARGOT2 function prediction tool [83,84], which was able to annotate grapevine protein sequences by blastp search against the UniProtKB database [85] (downloaded from January 2012) and by association of Gene Ontology biological process terms [86] weighted according to both semantic similarity relations and associated scores [83,84]. Annotation of differentially expressed genes and their distribution over 15 selected functional categories was compared with the entire grapevine transcriptome using GOstat statistical analysis 
[87]. For manual annotation, grapevine proteins were aligned against the Swiss Prot [88] database (downloaded from February 2012) using blastp, and the best hits (E-value lower than $1 \mathrm{E}^{-5}$ ) were used to select protein descriptions. The corresponding genes of the grapevine PN40024 genotype [89] were identified by blastp search against the $12 \times$ release [90] with an imposed E-value lower than $1 \mathrm{E}^{-20}$.

\section{Gene expression analysis by quantitative real-time RT-PCR} Total RNA was treated with DNase I (Invitrogen, Carlsbad, CA), and the first-strand cDNA was synthesized from $1.0 \mu \mathrm{g}$ of total RNA using Superscript III (Invitrogen) and oligo-dT. Reactions were carried out with Platinum SYBR Green qPCR SuperMix-UDG (Invitrogen) and specific primers (Additional file 11) using the Light Cycler 480 (Roche Diagnostics, Germany). The PCR conditions were: $50^{\circ} \mathrm{C}$ for $2 \mathrm{~min}$ and $95^{\circ} \mathrm{C}$ for $2 \mathrm{~min}$ as initial steps, followed by 50 cycles of $95^{\circ} \mathrm{C}$ for $15 \mathrm{~s}$ and $60^{\circ} \mathrm{C}$ for $1 \mathrm{~min}$. Each sample was examined in three technical replicates, and dissociation curves were analysed to verify the specificity of each amplification reaction. Light Cycler 480 SV1.5.0 software (Roche) was used to extract $\mathrm{Ct}$ values using the second derivative calculation [91], and LinReg software was used to calculate reaction efficiencies [92]. The relative expression of each gene was then calculated according to the Pfaffl equation [93] using control plants as the calibrator. The actin gene (glimmer.VV78X114914.6_2) was used as the constitutive gene for normalization $[4,52]$ because its expression was not significantly affected by the treatments (Additional file 12), and comparable results were obtained with VATP16 [94]. Mean expression and standard error of three biological replicates were calculated for each treatment.

\section{Additional files}

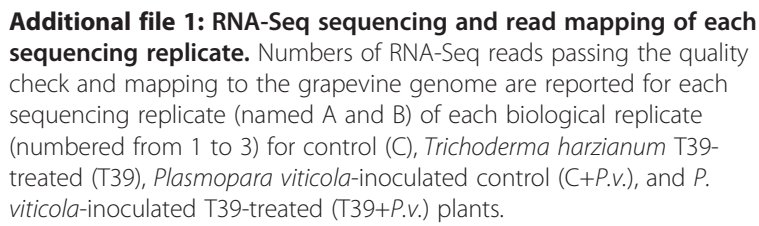

Additional file 2: RNA-Seq sequencing and coverage of the grapevine transcriptome for each biological and sequencing replicate. Total bases (Mbp) sequenced by RNA-Seq analysis and coverage of the grapevine transcriptome are reported for each sequencing replicate (named A and B) of each biological replicate (numbered from 1 to 3) for control (C), Trichoderma harzianum T39treated (T39), Plasmopara viticola-inoculated control (C+P.V.), and P. viticola-inoculated T39-treated (T39+P.v.) plants.

Additional file 3: Distribution of RNA-Seq sequences within the grapevine genome. (A) Proportions of reads mapping to unique locations (unique reads, yellow), reads mapping to multiple locations with 2-100 matches (multi-reads, red), and reads not mapping or ambiguously mapping to more than 100 locations (unused reads, white) to the grapevine genome. The mean number of sequenced reads and standard errors for three biological replicates are presented for each treatment. Percentages (\%) are calculated with respect to the total filtered reads. Unique reads and multi-reads were used for evaluating gene expression. (B) Proportions of reads mapping to grapevine genes (green) and to intergenic regions (red) of the grapevine genome. The mean number of sequenced reads and standard errors for three biological replicates are presented for each treatment. Percentages (\%) are calculated with respect to the total mapping reads. Grapevine treatments: control (C), Trichoderma harzianum T39-treated (T39), Plasmopara viticola-inoculated control (C+P.V.), and P. viticola-inoculated T39-treated (T39+P.V) plants.

Additional file 4: RNA-Seq reads mapping to grapevine genes. Numbers of RNA-Seq reads mapping to grapevine genes are reported for each sequencing replicate (named A and B) of each biological replicate (numbered from 1 to 3 ) for control (C), Trichoderma harzianum T39treated (T39), Plasmopara viticola-inoculated control (C+P.V.), and P. viticola-inoculated T39-treated (T39+P.v.) plants.

Additional file 5: Expressed grapevine genes and novel genes identified by RNA-Seq analysis. Numbers of expressed grapevine genes and novel genes with respect to the grapevine annotation are reported for each sequencing replicate (named A and B) of each biological replicate (numbered from 1 to 3 ) for control (C), Trichoderma harzianum T39-treated (T39), Plasmopara viticola-inoculated control (C+P. v.), and P. viticola-inoculated T39-treated (T39+P.v.) plants

Additional file 6: Expression levels of grapevine genes. Gene expression values (FPKM) and standard errors are reported for all $(33,514)$ Pinot Noir grapevine predicated genes [77] Release 3 [78] in control (C), Trichoderma harzianum T39-treated (T39), Plasmopara viticola-inoculated control (C+P.V.), and P. viticola-inoculated T39-treated (T39+P.V.) plants. Expression values in each biological replicate (numbered from 1 to 3 ) and each sequencing replicate (named A and B) are also listed.

Additional file 7: Correlations between sequencing replicates of RNA-Seq analysis. Comparison of the expression levels of all grapevine genes, expressed as fragments per kilobase of transcript per million fragments mapped (FPKM), in the two sequencing replicates (named $A$ and B) of (A) control (C) biological replicate no. 1; (B) C biological replicate no. 2 (B); (C) C biological replicate no. 3; (D) Trichoderma harzianum T39-treated (T39) biological replicate no. 1; (E) T39-treated biological replicate no. 2; (F) T39-treated biological replicate no. 3; (G) Plasmopara viticola-inoculated control (C+P.v.) biological replicate no. 1; (H) C+P.v. biological replicate no. 2; (I) C+P.V. biological replicate no. 3; (J) P. viticola-inoculated T39-treated plants (T39+P.v) biological replicate no. 1; (K) T39+P.v biological replicate no. 2; and (L) T39+P.v biological replicate no. 3.

Additional file 8: Principal component analysis of grapevine treatments. Principal component analysis (PCA) is based on the expression values (FPKM) of all grapevine genes for each sequencing replicate (named $\mathrm{A}$ and $\mathrm{B}$ ) of each biological replicate (numbered from 1 to 3) for control (C), Trichoderma harzianum T39-treated (T39), Plasmopara viticola-inoculated control (C+P.v.), and P. viticola-inoculated T39-treated (T39+P.v.) plants.

Additional file 9: Pearson's correlation coefficients between sequencing and biological replicates of RNA-Seq analysis. Pearson's correlation coefficients are based on the gene expression values (FPKM) of all grapevine genes in two sequencing replicates (A and B) of each biological replicate (numbered from 1 to 3) for control (C), Trichoderma harzianum T39-treated (T39), Plasmopara viticola-inoculated control (C+P. v.), and P. viticola-inoculated T39-treated (T39+P.v.) plants.

Additional file 10: Expression levels, clustering, and annotation results of differentially expressed genes. Gene expression values (FPKM) and standard errors are reported for differentially expressed $(7,024)$ genes in control $(C)$, Trichoderma harzianum T39-treated (T39), Plasmopara viticola-inoculated control (C+P.v.), and P. viticola-inoculated T39-treated (T39+P.V.) plants. Differentially expressed genes were identified by the DESeq package with a false discovery rate (FDR) of 5\% and a fold-change greater than two in at least one pairwise comparison. 
Fold-changes, clustering results, and functional annotation are reported for each gene.

\section{Additional file 11: Primer sequences of the grapevine genes} analysed by real-time RT-PCR. Forward and reverse primer sequences of real-time RT-PCR analysis are reported for 24 selected grapevine genes. Grapevine Actin (TC81781) [4, 52] and VATP16 (XM_002269086.1) [94] were used as constitutive genes for normalising the real-time RT-PCR data.

Additional file 12: Expression levels of grapevine genes that are members of the actin gene family. Gene expression values (FPKM) and standard errors are reported for grapevine genes belonging to the Actin gene family in control (C), Trichoderma harzianum T39-treated (T39), Plasmopara viticola-inoculated control (C+P.v.), and P. viticola-inoculated T39-treated (T39+P.v) plants.

\section{Competing interests}

The authors declare that they have no competing interests.

\section{Authors' contributions}

MP performed the grapevine treatments, P. viticola inoculations, RNA extractions, and real-time RT-PCR experiments. MP, MM, PF, and AF analysed the RNA-Seq sequences by mapping, gene expression estimation, and statistical analysis. MP, CM, RV, MD, and IP contributed to data interpretation and manuscript writing. MP, CM, MD, and IP conceived the study, designed the experiment, and coordinated all research activities. All authors read and approved the final manuscript.

\section{Acknowledgments}

This research was supported by the Post-Doc Project 2006 Resistevite, Envirochange and Accordo di programma funded by the Autonomous Province of Trento. The authors thank Dr. Yigal Elad (The Volcani Center, Bet Dagan, Israel) for supplying Trichodex and Dr. Laurent Farinelli (Fasteris SA, Plan-les-Ouates, Switzerland) for technical support with the RNA-Seq Illumina sequencing.

\section{Author details}

'IASMA Research and Innovation Centre, Fondazione Edmund Mach, Via E. Mach 1, 38010, San Michele all'Adige (TN), Italy. ${ }^{2}$ Dipartimento di Biotecnologie, Università degli Studi di Verona, Strada Le Grazie 15, 37134, Verona, Italy.

Received: 3 August 2012 Accepted: 13 November 2012

Published: 22 November 2012

\section{References}

1. Gessler C, Pertot I, Perazzolli M: Plasmopara viticola, the causal agent of downy mildew of grapes. Phytopathol Mediterr 2011, 50:3-44.

2. Pimentel D, Acquay H, Biltonen M, Rice P, Silva M, Nelson J, Lipner V, Giordano S, Horowitz A, D'Amore M: Environmental and economic costs of pesticide use. Bioscience 1992, 42:750-760.

3. Chen WJ, Delmotte F, Richard-Cervera S, Douence L, Greif C, Corio-Costet MF: At least two origins of fungicide resistance in grapevine downy mildew populations. Appl Environ Microbiol 2007, 73:5162-5172.

4. Polesani M, Bortesi L, Ferrarini A, Zamboni A, Fasoli M, Zadra C, Lovato A, Pezzotti M, Delledonne M, Polverari A: General and species-specific transcriptional responses to downy mildew infection in a susceptible (Vitis vinifera) and a resistant (V. riparia) grapevine species. BMC Genomics 2010, 11:117.

5. Polesani M, Desario F, Ferrarini A, Zamboni A, Pezzotti M, Kortekamp A, Polverari A: CDNA-AFLP analysis of plant and pathogen genes expressed in grapevine infected with Plasmopara viticola. BMC Genomics 2008, 9:142.

6. Wu J, Zhang Y, Zhang H, Huang H, Folta KM, Lu J: Whole genome wide expression profiles of Vitis amurensis grape responding to downy mildew by using Solexa sequencing technology. BMC Plant Biol 2010, 10:234.

7. Legay G, Marouf E, Berger D, Neuhaus JM, Mauch-Mani B, Slaughter A: Identification of genes expressed during the compatible interaction of grapevine with Plasmopara viticola; through suppression subtractive hybridization (SSH). Eur J Plant Pathol 2011, 129:281-301.
8. Malacarne G, Vrhovsek U, Zulini L, Cestaro A, Stefanini M, Mattivi F, Delledonne M, Velasco R, Moser C: Grapevine resistance to Plasmopara viticola is associated to stilbenoids accumulation and to specific transcriptional responses as revealed by metabolic and gene expression profiling of resistant and susceptible individuals in a segregating population. BMC Plant Biol 2011, 11:114

9. Hayes MA, Feechan A, Dry IB: Involvement of abscisic acid in the coordinated regulation of a stress-inducible hexose transporter (VvHT5) and a cell wall invertase in grapevine in response to biotrophic fungal infection. Plant Physiol 2010, 153:211-221.

10. Gamm M, Heloir MC, Bligny R, Vaillant-Gaveau N, Trouvelot S, Alcaraz G, Frettinger $P$, Clement C, Pugin A, Wendehenne D, Adrian M: Changes in carbohydrate metabolism in Plasmopara viticola infected grapevine leaves. Mol Plant Microbe Interact 2011, 24:1061-1073.

11. Kortekamp A: Expression analysis of defence-related genes in grapevine leaves after inoculation with a host and a non-host pathogen. Plant Physiol Biochem 2006, 44:58-67.

12. Casagrande K, Falginella L, Castellarin SD, Testolin R, Di Gaspero G: Defence responses in Rpv3-dependent resistance to grapevine downy mildew. Planta 2011, 234:1097-1109.

13. Figueiredo A, Monteiro F, Fortes AM, Bonow-Rex M, Zyprian E, Sousa L, Pais MS: Cultivar-specific kinetics of gene induction during downy mildew early infection in grapevine. Funct Integr Genomics 2012, 12:379-386

14. He M, Xu Y, Cao J, Zhu Z, Jiao Y, Wang Y, Guan X, Yang Y, Xu W, Fu Z: Subcellular localization and functional analyses of a PR10 protein gene from Vitis pseudoreticulata in response to Plasmopara viticola infection. Protoplasma 2012, 10.1007/s00709-012-0384-8.

15. Aziz A, Trotel-Aziz P, Dhuicq L, Jeandet P, Couderchet M, Vernet G: Chitosan oligomers and copper sulfate induce grapevine defense reactions and resistance to gray mold and downy mildew. Phytopathology 2006, 96:1188-1194

16. Aziz A, Poinssot B, Daire X, Adrian M, Bezier A, Lambert B, Joubert JM, Pugin $A$ : Laminarin elicits defense responses in grapevine and induces protection against Botrytis cinerea and Plasmopara viticola. Mol Plant Microbe Interact 2003, 16:1118-1128.

17. Trouvelot $S$, Varnier AL, Allègre M, Mercier L, Baillieul F, Arnould C, Gianinazzi-Pearson V, Klarzynski O, Joubert JM, Pugin A, Daire X: A beta-1,3 glucan sulfate induces resistance in grapevine against Plasmopara viticola through priming of defense responses, including HR-like cell death. Mol Plant Microbe Interact 2008, 21:232-243.

18. Allègre M, Héloir MC, Trouvelot $S$, Daire $X$, Pugin A, Wendehenne D, Adrian M: Are grapevine stomata involved in the elicitor-induced protection against downy mildew? Mol Plant Microbe Interact 2009, 22:977-986.

19. Hamiduzzaman MM, Jakab G, Barnavon L, Neuhaus JM, Mauch-Mani B: Beta-aminobutyric acid-induced resistance against downy mildew in grapevine acts through the potentiation of callose formation and jasmonic acid signaling. Mol Plant Microbe Interact 2005, 18:819-829.

20. Dubreuil-Maurizi C, Trouvelot S, Frettinger P, Pugin A, Wendehenne D, Poinssot B: Beta-aminobutyric acid primes an NADPH oxidase-dependent reactive oxygen species production during grapevine-triggered immunity. Mol Plant Microbe Interact 2010, 23:1012-1021.

21. Perazzolli M, Dagostin S, Ferrari A, Elad Y, Pertot I: Induction of systemic resistance against Plasmopara viticola in grapevine by Trichoderma harzianum T39 and benzothiadiazole. Biol Control 2008, 47:228-234.

22. Harm A, Kassemeyer H-H, Seibicke T, Regner F: Evaluation of chemical and natural resistance inducers against downy mildew (Plasmopara viticola) in grapevine. Am J Enol Vitic 2011, 62:184-192

23. Boubakri H, Wahab MA, Chong J, Bertsch C, Mliki A, Soustre-Gacougnolle I: Thiamine induced resistance to Plasmopara viticola in grapevine and elicited host-defense responses, including HR like-cell death. Plant Physiol Biochem 2012, 57:120-133.

24. Godard S, Slacanin I, Viret O, Gindro K: Induction of defence mechanisms in grapevine leaves by emodin- and anthraquinone-rich plant extracts and their conferred resistance to downy mildew. Plant Physiol Biochem 2009, 47:827-837.

25. Thuerig B, Binder A, Boller T, Guyer U, Jiménez S, Rentsch C, Tamm L: An aqueous extract of the dry mycelium of Penicillium chrysogenum induces resistance in several crops under controlled and field conditions. Eur J Plant Pathol 2006, 114:185-197. 
26. Segarra G, van der Ent S, Trillas I, Pieterse CMJ: MYB72, a node of convergence in induced systemic resistance triggered by a fungal and a bacterial beneficial microbe. Plant Biol 2009, 11:90-96

27. Shoresh M, Harman GE, Mastouri F: Induced systemic resistance and plant responses to fungal biocontrol agents. Annu Rev Phytopathol 2010, 48:21-43.

28. Bailey BA, Bae H, Strem MD, Roberts DP, Thomas SE, Crozier J, Samuels GJ, Choi IY, Holmes KA: Fungal and plant gene expression during the colonization of cacao seedlings by endophytic isolates of four Trichoderma species. Planta 2006, 224:1449-1464.

29. Alfano G, Ivey ML, Cakir C, Bos Jl, Miller SA, Madden LV, Kamoun S, Hoitink HA: Systemic modulation of gene expression in tomato by Trichoderma hamatum 382. Phytopathology 2007, 97:429-437.

30. Brotman Y, Lisec J, Michaël M, Chet I, Willmitzer L, Viterbo A: Transcript and metabolite analysis of the Trichoderma-induced systemic resistance response to Pseudomonas syringae in Arabidopsis thaliana. Microbiology 2012, 158:139-146.

31. Mathys J, De Cremer K, Timmermans P, Van Kerckhove S, Lievens B, Vanhaecke M, Cammue BP, De Coninck B: Genome-wide characterization of ISR induced in Arabidopsis thaliana by Trichoderma hamatum T382 against Botrytis cinerea infection. Front Plant Sci 2012, 3:108.

32. Morán-Diez E, Rubio B, Domínguez S, Hermosa R, Monte E, Nicolás C: Transcriptomic response of Arabidopsis thaliana after $24 \mathrm{~h}$ incubation with the biocontrol fungus Trichoderma harzianum. J Plant Physiol 2012, 169:614-620.

33. Pieterse CMJ, Leon-Reyes A, Van der Ent S, Van Wees SC: Networking by small-molecule hormones in plant immunity. Nat Chem Biol 2009, 5:308-316.

34. Pieterse CMJ, Van Pelt JA, Ton J, Parchmann S, Mueller MJ, Buchala AJ, Métraux JP, Van Loon LC: Rhizobacteria-mediated induced systemic resistance (ISR) in Arabidopsis requires sensitivity to jasmonate and ethylene but is not accompanied by an increase in their production. Physiol Mol Plant Pathol 2000, 57:123-134.

35. Van Wees SCM, Van der Ent S, Pieterse CMJ: Plant immune responses triggered by beneficial microbes. Curr Opin Plant Biol 2008, 11:443-448.

36. van der Ent S, Koornneef A, Ton J, Pieterse CMJ: Induced resistanceorchestrating defence mechanisms through crosstalk and priming. In Annual plant reviews - molecular aspects of plant disease resistance, 34. Edited by Parker JE. Oxford, UK: Wiley-Blackwell; 2009:334-370.

37. Conrath U, Beckers GJM, Flors V, Garcia-Agustin P, Jakab G, Mauch F, Newman MA, Pieterse CMJ, Poinssot B, Pozo MJ, Pugin A, Schaffrath U, Ton J, Wendehenne D, Zimmerli L, Mauch-Mani B: Priming: getting ready for battle. Mol Plant Microbe Interact 2006, 19:1062-1071.

38. Verhagen BW, Glazebrook J, Zhu T, Chang HS, van Loon LC, Pieterse CMJ: The transcriptome of rhizobacteria-induced systemic resistance in Arabidopsis. Mol Plant Microbe Interact 2004, 17:895-908.

39. van Hulten M, Pelser M, van Loon LC, Pieterse CMJ, Ton J: Costs and benefits of priming for defense in Arabidopsis. Proc Natl Acad Sci USA 2006, 103:5602-5607.

40. Walters DR, Heil M: Costs and trade-offs associated with induced resistance. Physiol Mol Plant Pathol 2007, 71:3-17.

41. Beckers GJM, Conrath U: Priming for stress resistance: from the lab to the field. Curr Opin Plant Biol 2007, 10:425-431.

42. Perazzolli M, Raoatti B, Bozza E, Pertot I: Trichoderma harzianum T39 induces resistance against downy mildew by priming for defence without costs for grapevine. Biol Control 2011, 58:74-82.

43. Beckers GJ, Jaskiewicz M, Liu Y, Underwood WR, He SY, Zhang S, Conrath U: Mitogen-activated protein kinases 3 and 6 are required for full priming of stress responses in Arabidopsis thaliana. Plant Cell 2009, 21:944-953.

44. Verhagen BW, Trotel-Aziz P, Couderchet M, Hofte M, Aziz A: Pseudomonas spp.-induced systemic resistance to Botrytis cinerea is associated with induction and priming of defence responses in grapevine. J Exp Bot 2010, 61:249-260

45. Mortazavi A, Williams BA, McCue K, Schaeffer L, Wold B: Mapping and quantifying mammalian transcriptomes by RNA-Seq. Nat Methods 2008, 5:621-628.

46. Trapnell C, Williams BA, Pertea G, Mortazavi A, Kwan G, van Baren MJ, Salzberg SL, Wold BJ, Pachter L: Transcript assembly and quantification by RNA-Seq reveals unannotated transcripts and isoform switching during cell differentiation. Nat Biotechnol 2010, 28:511-515.
47. Anders S, Huber W: Differential expression analysis for sequence count data. Genome Biol 2010, 11:R106.

48. Mizuno $H$, Kawahara $Y$, Sakai $H$, Kanamori $H$, Wakimoto $H$, Yamagata $H$, Oono Y, Wu J, Ikawa H, Itoh T, Matsumoto T: Massive parallel sequencing of mRNA in identification of unannotated salinity stress-inducible transcripts in rice (Oryza sativa L.). BMC Genomics 2010, 11:683.

49. Zenoni S, Ferrarini A, Giacomelli E, Xumerle L, Fasoli M, Malerba G, Bellin D, Pezzotti M, Delledonne M: Characterization of transcriptional complexity during berry development in Vitis vinifera using RNA-Seq. Plant Physiol 2010, 152:1787-1795.

50. Chong J, Le Henanff G, Bertsch C, Walter B: Identification, expression analysis and characterization of defense and signaling genes in Vitis vinifera. Plant Physiol Biochem 2008, 46:469-481.

51. Mzid R, Marchive C, Blancard D, Deluc L, Barrieu F, Corio-Costet MF, Drira N, Hamdi S, Lauvergeat V: Overexpression of VvWRKY2 in tobacco enhances broad resistance to necrotrophic fungal pathogens. Physiol Plant 2007, 131:434-447.

52. Milli A, Cecconi D, Bortesi L, Persi A, Rinalducci S, Zamboni A, Zoccatelli G, Lovato A, Zolla L, Polverari A: Proteomic analysis of the compatible interaction between Vitis vinifera and Plasmopara viticola. J Proteomics 2012, 75:1284-1302.

53. Shoresh M, Gal-On A, Leibman D, Chet I: Characterization of a mitogenactivated protein kinase gene from cucumber required for trichodermaconferred plant resistance. Plant Physiol 2006, 142:1169-1179.

54. Moreno CA, Castillo F, González A, Bernal D, Jaimes Y, Chaparro M, González C, Rodriguez F, Restrepo S, Cotes AM: Biological and molecular characterization of the response of tomato plants treated with Trichoderma koningiopsis. Physiol Mol Plant Pathol 2009, 74:111-120.

55. Palmieri MC, Perazzolli M, Matafora V, Moretto M, Bachi A, Pertot I: Proteomic analysis of grapevine resistance induced by Trichoderma harzianum $T 39$ reveals specific defence pathways activated against downy mildew. J Exp Bot 2012, 63:6237-6251.

56. Lukasik E, Takken FL: STANDing strong, resistance proteins instigators of plant defence. Curr Opin Plant Biol 2009, 12:427-436.

57. Thilmony R, Underwood W, He SY: Genome-wide transcriptional analysis of the Arabidopsis thaliana interaction with the plant pathogen Pseudomonas syringae pv. tomato DC3000 and the human pathogen Escherichia coli 0157:H7. Plant J 2006, 46:34-53.

58. Korolev N, Rav David D, Elad Y: The role of phytohormones in basal resistance and Trichoderma-induced systemic resistance to Botrytis cinerea in Arabidopsis thaliana. BioControl 2008, 53:667-683.

59. Van der Ent S, Verhagen BW, Van Doorn R, Bakker D, Verlaan MG, Pel MJ, Joosten RG, Proveniers MC, Van Loon LC, Ton J, Pieterse CM: MYB72 is required in early signaling steps of rhizobacteria-induced systemic resistance in Arabidopsis. Plant Physiol 2008, 146:1293-1304.

60. Wu A, Allu AD, Garapati P, Siddiqui H, Dortay H, Zanor Ml, Asensi-Fabado MA, Munne-Bosch S, Antonio C, Tohge T, Fernie AR, Kaufmann K, Xue GP, Mueller-Roeber B, Balazadeh S: JUNGBRUNNEN1, a reactive oxygen species-responsive NAC transcription factor, regulates longevity in Arabidopsis. Plant Cell 2012, 24:482-506.

61. Mohr TJ, Mammarella ND, Hoff T, Woffenden BJ, Jelesko JG, McDowell JM: The Arabidopsis downy mildew resistance gene RPP8 is induced by pathogens and salicylic acid and is regulated by $\mathrm{W}$ box cis elements. Mol Plant Microbe Interact 2010, 23:1303-1315.

62. Conrath U: Molecular aspects of defence priming. Trends Plant Sci 2011, 16:524-531.

63. Jaskiewicz M, Conrath U, Peterhansel C: Chromatin modification acts as a memory for systemic acquired resistance in the plant stress response. EMBO Rep 2011, 12:50-55.

64. Slaughter A, Daniel X, Flors V, Luna E, Hohn B, Mauch-Mani B: Descendants of primed Arabidopsis plants exhibit resistance to biotic stress. Plant Physiol 2012, 158:835-843.

65. Bultreys A, Trombik T, Drozak A, Boutry M: Nicotiana plumbaginifolia plants silenced for the ATP-binding cassette transporter gene NpPDR1 show increased susceptibility to a group of fungal and oomycete pathogens. Mol Plant Pathol 2009, 10:651-663.

66. Kamoun S: A catalogue of the effector secretome of plant pathogenic oomycetes. Annu Rev Phytopathol 2006, 44:41-60.

67. Stassen JH, Van den Ackerveken G: How do oomycete effectors interfere with plant life? Curr Opin Plant Biol 2011, 14:407-414. 
68. Birch PR, Rehmany AP, Pritchard L, Kamoun S, Beynon JL: Trafficking arms: oomycete effectors enter host plant cells. Trends Microbiol 2006, 14:8-11.

69. Mestre P, Piron MC, Merdinoglu D: Identification of effector genes from the phytopathogenic oomycete Plasmopara viticola through the analysis of gene expression in germinated zoospores. Fungal Biol 2012, 116:825-835.

70. Díez-Navajas AM, Wiedemann-Merdinoglu S, Greif C, Merdinoglu D: Nonhost versus host resistance to the grapevine downy mildew, Plasmopara viticola, studied at the tissue level. Phytopathology 2008, 98:776-780

71. Malacarne G, Perazzolli M, Cestaro A, Sterck L, Fontana P, Van de Peer Y, Viola R, Velasco R, Salamini F: Deconstruction of the (paleo)polyploid grapevine genome based on the analysis of transposition events involving NBS resistance genes. PLoS One 2012, 7:e29762.

72. Schnable JC, Springer NM, Freeling M: Differentiation of the maize subgenomes by genome dominance and both ancient and ongoing gene loss. Proc Natl Acad Sci USA 2011, 108:4069-4074.

73. Perazzolli M, Bampi F, Faccin S, Moser M, De Luca F, Ciccotti AM, Velasco R Gessler C, Pertot I, Moser C: Armillaria mellea induces a set of defense genes in grapevine roots and one of them codifies a protein with antifungal activity. Mol Plant Microbe Interact 2010, 23:485-496.

74. EPPO: European and Mediterranean Plant Protection Organization (EPPO): Guidelines for the efficacy evaluation of fungicides: Plasmopara viticola. EPPO Bull 2001, 31:313-317.

75. Unger S, Buche C, Boso S, Kassemeyer HH: The course of colonization of two different Vitis genotypes by Plasmopara viticola indicates compatible and incompatible host-pathogen interactions. Phytopathology 2007, 97:780-786.

76. Sequence read archive (SRA). http://www.ncbi.nlm.nih.gov/sra/.

77. Velasco R, Zharkikh A, Troggio M, Cartwright DA, Cestaro A, Pruss D, Pindo M, Fitzgerald LM, Vezzulli S, Reid J, Malacarne G, lliev D, Coppola G, Wardell B, Micheletti D, Macalma T, Facci M, Mitchell JT, Perazzolli M, Eldredge G, Gatto P, Oyzerski R, Moretto M, Gutin N, Stefanini M, Chen Y, Segala C, Davenport C, Dematte L, Mraz A, et al: A high quality draft consensus sequence of the genome of a heterozygous grapevine variety. PLoS One 2007, 2:e1326.

78. Pinot noir grapevine genome browser. http://genomics.research.iasma.it.

79. Trapnell C, Pachter L, Salzberg SL: TopHat: discovering splice junctions with RNA-Seq. Bioinformatics 2009, 25:1105-1111.

80. Roberts A, Trapnell C, Donaghey J, Rinn JL, Pachter L: Improving RNA-Seq expression estimates by correcting for fragment bias. Genome Biol 2011, 12:R22.

81. Roberts A, Pimentel H, Trapnell C, Pachter L: Identification of novel transcripts in annotated genomes using RNA-Seq. Bioinformatics 2011 27:2325-2329.

82. Saeed Al, Bhagabati NK, Braisted JC, Liang W, Sharov V, Howe EA, Li J, Thiagarajan M, White JA, Quackenbush J: TM4 microarray software suite. Methods Enzymol 2006, 411:134-193.

83. Fontana P, Cestaro A, Velasco R, Formentin E, Toppo S: Rapid annotation of anonymous sequences from genome projects using semantic similarities and a weighting scheme in gene ontology. PLoS One 2009, 4:e4619.

84. Falda M, Toppo S, Pescarolo A, Lavezzo E, Di Camillo B, Facchinetti A, Cilia E, Velasco R, Fontana P: Argot2: a large scale function prediction too relying on semantic similarity of weighted Gene Ontology terms. BMC Bioinforma 2012, 13(Suppl 4):S14.

85. UniProt. http://www.uniprot.org/.

86. The gene ontology. http://www.geneontology.org/.

87. Beissbarth T, Speed TP: GOstat: find statistically overrepresented Gene Ontologies within a group of genes. Bioinformatics 2004, 20:1464-1465.

88. ExPASy. http://www.expasy.ch/.

89. Jaillon O, Aury JM, Noel B, Policriti A, Clepet C, Casagrande A, Choisne N, Aubourg S, Vitulo N, Jubin C, Vezzi A, Legeai F, Hugueney P, Dasilva C, Horner D, Mica E, Jublot D, Poulain J, Bruyere C, Billault A, Segurens B, Gouyvenoux M, Ugarte E, Cattonaro F, Anthouard V, Vico V, Del Fabbro C, Alaux M, Di Gaspero G, Dumas V, et al: The grapevine genome sequence suggests ancestral hexaploidization in major angiosperm phyla. Nature 2007, 449:463-467.

90. Grape genome browser. http://www.genoscope.cns.fr/externe/ GenomeBrowser/Vitis/.
91. Luu-The V, Paquet N, Calvo E, Cumps J: Improved real-time RT-PCR method for high-throughput measurements using second derivative calculation and double correction. Biotechniques 2005, 38:287-293.

92. Ruijter JM, Ramakers C, Hoogaars WM, Karlen Y, Bakker O, van den Hoff MJ, Moorman AF: Amplification efficiency: linking baseline and bias in the analysis of quantitative PCR data. Nucleic Acids Res 2009, 37:e45.

93. Pfaffl MW: A new mathematical model for relative quantification in realtime RT-PCR. Nucleic Acids Res 2001, 29:e45.

94. Gamm M, Héloir MC, Kelloniemi J, Poinssot B, Wendehenne D, Adrian M: Identification of reference genes suitable for qRT-PCR in grapevine and application for the study of the expression of genes involved in pterostilbene synthesis. Mol Genet Genomics 2011, 285:273-285.

\section{doi:10.1186/1471-2164-13-660}

Cite this article as: Perazzolli et al:: Downy mildew resistance induced by Trichoderma harzianum T39 in susceptible grapevines partially mimics transcriptional changes of resistant genotypes. BMC Genomics 2012 13:660.

\section{Submit your next manuscript to BioMed Central and take full advantage of:}

- Convenient online submission

- Thorough peer review

- No space constraints or color figure charges

- Immediate publication on acceptance

- Inclusion in PubMed, CAS, Scopus and Google Scholar

- Research which is freely available for redistribution 